\title{
含三氟甲基吡啶结构的酰胺类衍生物的合成、表征及生物活性研究
}

\author{
何 凤郭声金鍂惐丽张仁凤吴 剑* \\ (贵州大学绿色农药与农业生物工程国家重点实验室培育基地 教育部绿色农药与 \\ 农业生物工程重点实验室 贵阳 550025)
}

\begin{abstract}
摘要 合成了一系列含三氟甲基吡啶结构的酰胺衍生物, 并测试了它们对水稻白叶枯病菌(Xanthomonas oryzae pv. oryzae)、柑滳溃疡病菌(Xanthomonas axonopodis pv. citri)、烟草青枯病菌(Ralstonia solanacearum)的体外抑菌活性和对 小菜蛾(Plutella xylostella) 的杀虫活性. 结果表明, 部分化合物表现出优异的抑菌活性. 如 2-((3-氯-5-(三氟甲基)吡啶-2基)氧基)- $N$-(4-氟-2-甲基苯基)乙酰胺 (6d) 对水稻白叶枯病菌的 $\mathrm{EC}_{50}$ 值为 $54.1 \mathrm{mg} \cdot \mathrm{L}^{-1}$, 低于叶枯唑 $\left(\mathrm{EC}_{50}=59.6 \mathrm{mg} \cdot \mathrm{L}^{-1}\right.$ ) 和噻菌酮 $\left(\mathrm{EC}_{50}=86.3 \mathrm{mg} \cdot \mathrm{L}^{-1}\right)$. 2-((3-氯-5-(三氟甲基)吡啶-2-基)氧基)- $N$-(4-氟-3(三氟甲基)苯基)乙酰胺(6h) 和 2-((3-氯5-(三氟甲基)吡啶-2-基)氧基)- $N$-(5,6-二氯吡啶-3-基)乙酰胺(6z)对柑滳溃疡病菌的抑制活性( $\mathrm{EC}_{50}$ 值为 51.2 和 $60.7 \mathrm{mg} \cdot$ $\left.\mathrm{L}^{-1}\right)$ 均高于商品药剂叶枯唑 $\left(\mathrm{EC}_{50}=76.3 \mathrm{mg} \cdot \mathrm{L}^{-1}\right)$ 和噻菌酮 $\left(\mathrm{EC}_{50}=101.7 \mathrm{mg} \cdot \mathrm{L}^{-1}\right) . \mathrm{N}$ - $(2$-氯-4-氟苯基)-2-( (3-氯-5-(三氟甲 基)吡啶-2-基)氧基)乙酰胺(6e)对烟草青枯病菌的抑菌活性略高于噻菌酮 $\left(\mathrm{EC}_{50}=79.0 \mathrm{mg} \cdot \mathrm{L}^{-1}\right), \mathrm{EC}_{50}$ 值为 $74.9 \mathrm{mg} \cdot \mathrm{L}^{-1}$. 此 外, 化合物 6e 和 2-((3-氯-5-(三氟甲基)吡啶-2-基)氧基)- $N$-(3-异丙基苯基)乙酰胺(6k)在浓度为 $500 \mathrm{mg} \cdot \mathrm{L}^{-1}$ 时表现出中等 程度的杀小菜蛾活性.
\end{abstract}

关键词 三氟甲基吡啶; 酰胺衍生物; 合成; 抗细菌活性; 杀虫活性

\section{Synthesis, Characterization, and Biological Activity of Novel Amide Derivatives Containing Trifluoromethylpyridine Moieties}

\author{
He, Feng Guo, Shengxin Dai, Ali Zhang, Renfeng Wu, Jian* \\ (State Key Laboratory Breeding Base of Green Pesticide and Agricultural Bioengineering, Key Laboratory of Green \\ Pesticide and Agricultural Bioengineering, Ministry of Education, Guizhou University, Guiyang 550025)
}

\begin{abstract}
A series of novel trifluoromethylpyridine amide derivatives were synthesized and evaluated in vitro for their antibacterial activity against Xanthomonas oryzae pv. oryzae (Xoo), Xanthomonas axonopodis pv. citri (Xac), and Ralstonia solanacearum, and insecticidal activity against Plutella xylostella. Some of the compounds showed promising antibacterial activity. In particular, the half maximal effective concentration (EC50) of 2-((3-chloro-5-(trifluoromethyl)pyridin-2-yl)oxy)- $N$-(4-fluoro2-methylphenyl)acetamide (6d) was $54.1 \mathrm{mg} \cdot \mathrm{L}^{-1}$ against $X o o$, which was slightly lower than the bismerthiazol $\left(59.6 \mathrm{mg} \bullet \mathrm{L}^{-1}\right)$ and thiodiazole copper $\left(86.3 \mathrm{mg} \cdot \mathrm{L}^{-1}\right)$ positive controls. 2-((3-Chloro-5-(trifluoromethyl)pyridin-2-yl)oxy)- $N$-(4-fluoro-3(trifluoromethyl)phenyl)acetamide (6h) and 2-((3-chloro-5-(trifluoro-methyl)pyridin-2-yl)oxy)- $N$-(5,6-dichloropyridin-3-yl)acetamide (6z) exhibited much higher activity against $X a c\left(E_{50} 51.2\right.$ and $60.7 \mathrm{mg} \cdot \mathrm{L}^{-1}$, respectively) than bismerthiazol $(76.3$ $\left.\mathrm{mg} \cdot \mathrm{L}^{-1}\right)$ and thiodiazole copper $\left(101.7 \mathrm{mg} \cdot \mathrm{L}^{-1}\right) . \mathrm{N}$-(2-Chloro-4-fluorophenyl)-2-((3-chloro-5-(trifluoromethyl)pyridin-2-yl)oxy)acetamide (6e) showed good bioactivity against $R$. solanacearum with an $\mathrm{EC}_{50}\left(74.9 \mathrm{mg} \cdot \mathrm{L}^{-1}\right)$ lower than thiodiazole copper $\left(79.0 \mathrm{mg} \bullet \mathrm{L}^{-1}\right)$. 6e and 2-((3-chloro-5-(trifluoromethyl)pyridin-2-yl)oxy)- $N$-(3-isopropylphenyl)acetamide $(6 \mathbf{k})$ at $500 \mathrm{mg} \bullet$ $\mathrm{L}^{-1}$ exhibited moderate insecticidal activity against $P$. xylostella.
\end{abstract}

Keywords trifluoromethylpyridine; amide derivatives; synthesis; antibacterial activity; insecticidal activity

\section{Introduction}

Pathogens, including bacteria, cause major reductions in the yields of cash crops around the world. ${ }^{[1-3]}$ For example, Xanthomonas oryzae pv. oryzae (Xoo) causes one of the most destructive bacterial diseases of plants, leading to the

* Corresponding author. E-mail: jwu6@gzu.edu.cn

Received January 26, 2021; revised March 15, 2021; published online May 23, 2021.

Project supported by the National Natural Science Foundation of China (Nos. 21762012, 32072445, 21562012), the Program of Introducing Talents to Chinese Universities (111 Program, No. D20023), and the Science \& Technology Planning Project of Guizhou Province (Nos. [2017]1402, [2017]5788).

国家自然科学基金(Nos. 21762012, 32072445, 21562012)、高等学校学科创新引智计划(111 计划, No. D20023)及贵州省科技技术基金(Nos. 黔科合 [2017]1402, 黔科合[2017]5788)资助项目. 
loss of $80 \%$ of the annual rice yield. ${ }^{[4-6]}$ Xanthomonas axonopodis pv. citri $(\mathrm{Xac})$ causes citrus canker, possibly the most destructive disease in citrus plants. It is difficult to control once plants are infected and has a serious impact on the entire citrus industry. ${ }^{[7-9]}$ Ralstonia solanacearum ( $R$. solanacearum) causes tobacco wilt, a destructive disease leading to serious economic losses. ${ }^{[10]}$ Pesticides remain the primary intervention for controlling crop diseases. ${ }^{[11]}$ However, with the emergence of drug resistance and cross-resistance their efficacy has gradually decreased and there is still a lack of effective bactericides. ${ }^{[12]}$ Despite the ongoing threat of bacterial diseases and this lack of bactericides, effective prevention and extermination methods to manage these destructive diseases, particularly citrus canker and rice bacterial leaf blight, ${ }^{[13-14]}$ have still not been found. There is an urgent need to develop new environmentally friendly plant bactericides with novel modes of action, high activity, and low risk profiles.

Fluorine-containing compounds play an important role in pesticide chemistry, peculiarly in crop protection. ${ }^{[15]}$ The trifluoromethylpyridine skeleton is the most widely used fluorinated heterocycle in fluorinated pesticide design. ${ }^{[16-17]}$ For decades, trifluoromethylpyridine-containing compounds have exhibited promising crop protection effects via their antifungal, ${ }^{[18-20]}$ antiviral, ${ }^{[21]}$ insecticidal, ${ }^{[22-24]}$ and antibacterial activities. ${ }^{[25]}$ Recently, Wu et al. ${ }^{[26]}$ reported some trifluoromethylpyridine derivatives with good antibacterial activities against the invasive phytopathogenic bacteria Xoo, Xac, and $R$. solanacearum. Some small molecules containing the trifluoromethylpyridine moiety have shown good activity and have been commercialized. ${ }^{[27]}$ Consequently, trifluoromethylpyridine-containing derivatives are a hot topic in drug design.

Amide derivatives are also widely used in crop production, due to their insecticidal, antibacterial, and other biological activities. ${ }^{[28-29]}$ In 2017, using the insecticide tebufenpyrad as the lead compound, pyrazole amide derivatives with promising insecticidal activity against Oriental armyworm, Aphis medicaginis, and Tetranychus cinnabarinus were reported by Hong and co-workers. ${ }^{[30]}$ In 2020 a series of $\alpha$-ketoamide derivatives displaying excellent bactericidal activity against Xoo, Xac, and $R$. solanacearum were reported. ${ }^{[31]}$

The ether moiety has also been widely studied in pesticide design and used as an effective linker. ${ }^{[32]}$ Several ether-containing compounds have been developed for the control of Xoo, Xac, and $R$. solanacearum. ${ }^{[32-33]}$
Considering the above, this study combined trifluoromethylpyridine and amide structures (the substructures of fluopicolide and fluopyram, Figure 1) via an ether linker to produce new trifluoromethylpyridine derivatives bearing an amide. Antibacterial activity against Xoo, Xac and $R$. solanacearum, was then evaluated. Insecticidal activity against $P$. xylostella was also investigated. This study demonstrates that some of these novel compounds display promising antibacterial activity and moderate insecticidal activity.

\section{Results and discussion}

\subsection{Chemistry}

The general procedure for the preparation of the target compounds is shown in Scheme 1. The target compounds $\mathbf{6 a} \sim \mathbf{6 a b}$ were synthesized using 3-chloro-5-(trifluoromethyl)pyridin-2-ol and ethyl 2-chloroacetate as starting materials, with the formation of two intermediates ( 3 and 4). Intermediate 3 was prepared according to our previous work, ${ }^{[34]}$ then hydrolyzed under alkaline conditions to produce intermediate 4 . Intermediate 4 reacted with aniline in the presence of phosphorus oxychloride and triethylamine in $N, N$-dimethylformamide (DMF) to give target compounds $\mathbf{6 a} \sim \mathbf{6 a b} .^{[35]}$ Using DMSO- $d_{6}$ as the solvent, 6a $\sim 6 \mathbf{a b}$ were characterized by ${ }^{1} \mathrm{H} \mathrm{NMR},{ }^{13} \mathrm{C} \mathrm{NMR}$, and ${ }^{19}$ F NMR spectroscopy.

\subsection{Evaluation of antibacterial activity in vitro}

In vitro bio-assays using Xoo, Xac, and $R$. Solanacearum followed a previously reported method. ${ }^{[36-37]}$ Bismerthiazol (BT) and thiodiazole copper (TC), two bactericides registered for treatment of bacterial diseases, were used as positive control treatments. ${ }^{[36-37]}$ Table 1 shows that some of the target compounds exhibited higher antibacterial activity against Xoo, Xac, and $R$. Solanacearum than BT and TC when applied at 50 and $100 \mathrm{mg} \cdot \mathrm{L}^{-1}$. For example, at 100 $\mathrm{mg} \cdot \mathrm{L}^{-1}$, compound $\mathbf{6 d}$ ( $\mathrm{R}=4$-fluoro-2-methylphenyl) showed $62 \%$ activity against Xoo, which was slightly higher than both BT (59\%) and TC (56\%). Interestingly, the preliminary study of the structure-activity relationship (SAR) showed that the position of the substituent group on the aryl benzene is a significant factor affecting antibacterial activity against Xoo. For example, 6d ( $\mathrm{R}=4$-fluoro2-methylphenyl $)>\mathbf{6 a}(\mathrm{R}=2$-fluoro-4-methyl- phenyl $)>\mathbf{6 0}$ $(\mathrm{R}=2$-fluoro-5-methylphenyl) $>\mathbf{6 p}(\mathrm{R}=5$-fluoro-2-methyl phenyl); while 6y $(\mathrm{R}=2,6$-difluorophenyl $)>\mathbf{6 n}(\mathrm{R}=$

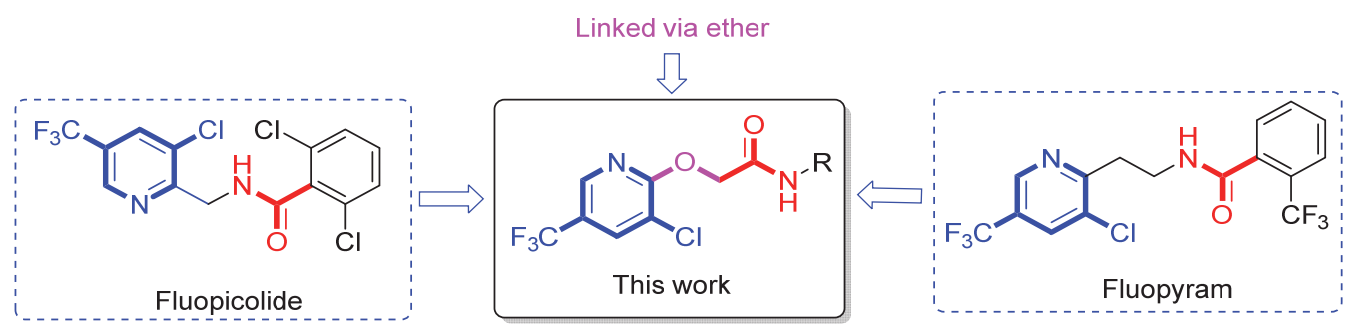

Figure 1 Design of the target compound 


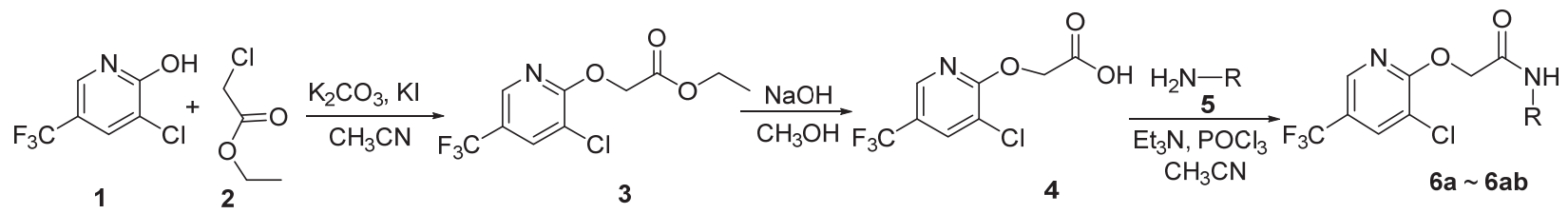

6a: $R=$ 2-fluoro-4-methylphenyl; 6d: R = 4-fluoro-2-methylphenyl; 6g: $\mathrm{R}=$ 2-chloro-6-methylphenyl; 6j: $\mathrm{R}=$ 4-chloro-2-methylphenyl; $6 \mathrm{~m}: \mathrm{R}=4$-(trifluoromethyl)phenyl ; 6p: $R=5$-fluoro-2-methylphenyl; 6s: R = 4-bromo-2-chlorophenyl; 6v: $\mathrm{R}=4$-(difluoromethoxy)phenyl; 6y: $R=2,6$-difluorophenyl; 6ab: $R=6$-trifluoromethyl)pyridin-3-yl 6b: $\mathrm{R}=3$-chloro-2-methylphenyl; 6e: $R=2$-chloro-4-fluorophenyl; 6h: $R=4-$ fluoro-3-(trifluoromethyl)phenyl; 6k: $\mathrm{R}=3$-isopropylphenyl; 6n: $R=2,4$-difluorophenyl; 6q: $R=3,5$-difluorophenyl; 6t: $\mathrm{R}=3$-methoxy-5-(trifluoromethyl)phenyl; 6w: R = 3,4-dimethoxyphenyl; 6z: $R=5,6$-dichloropyridin-3-yl; 6c: $R=4-$ methoxy-2-methylphenyl; 6f: $\mathrm{R}=4$-(trifluoromethoxy) phenyl; 6i: $\mathrm{R}=4$-chloro-3-fluorophenyl; 6I: $R=3,4$-difluorophenyl; 6o: $\mathrm{R}=$ 2-fluoro-5-methylphenyl; 6r: R = 4-bromo-2-methylphenyl;

6u: $R=2,4,5$-trifluorophenyl; 6x: $R=4$-bromo-3-fluorophenyl; 6aa: $R=2,6$-dichloropyridin-3-yl;

Scheme 1 Synthetic route to target compounds $6 \mathbf{a}$ to $\mathbf{6 a b}$

Table 1 Antibacterial activities of target compounds 6a to $6 \mathbf{a b}, \mathrm{BT}$ and $\mathrm{TC}^{a}$

\begin{tabular}{|c|c|c|c|c|c|c|}
\hline \multirow{2}{*}{ Compd. } & \multicolumn{2}{|c|}{ Хоо } & \multicolumn{2}{|c|}{$X a c$} & \multicolumn{2}{|c|}{ R. solanacearum } \\
\hline & $100 \mathrm{mg} \cdot \mathrm{L}^{-1}$ & $50 \mathrm{mg} \cdot \mathrm{L}^{-1}$ & $100 \mathrm{mg} \cdot \mathrm{L}^{-1}$ & $50 \mathrm{mg} \cdot \mathrm{L}^{-1}$ & $100 \mathrm{mg} \cdot \mathrm{L}^{-1}$ & $50 \mathrm{mg} \cdot \mathrm{L}^{-1}$ \\
\hline $6 a$ & $26.7 \pm 0.4$ & $20.6 \pm 4.5$ & $47.5 \pm 0.6$ & $38.5 \pm 1.8$ & $44.1 \pm 0.9$ & $28.4 \pm 3.0$ \\
\hline $6 b$ & $31.6 \pm 2.6$ & $24.8 \pm 1.1$ & $48.3 \pm 1.7$ & $40.9 \pm 1.2$ & $26.2 \pm 4.0$ & $22.5 \pm 1.1$ \\
\hline $6 c$ & $33.0 \pm 1.1$ & $22.2 \pm 4.1$ & $38.2 \pm 1.6$ & $27.0 \pm 1.7$ & $31.4 \pm 4.4$ & $22.0 \pm 4.2$ \\
\hline 6d & $61.7 \pm 3.4$ & $47.3 \pm 1.0$ & $41.3 \pm 2.1$ & $37.4 \pm 2.6$ & $18.0 \pm 2.5$ & $14.6 \pm 3.1$ \\
\hline $6 e$ & $32.8 \pm 3.1$ & $27.4 \pm 1.9$ & $44.2 \pm 3.8$ & $36.0 \pm 2.8$ & $58.5 \pm 2.4$ & $41.2 \pm 1.6$ \\
\hline $6 f$ & $39.1 \pm 3.0$ & $27.6 \pm 4.3$ & $48.3 \pm 2.6$ & $35.3 \pm 3.8$ & $43.3 \pm 2.9$ & $28.0 \pm 3.6$ \\
\hline $6 \mathrm{~g}$ & $32.1 \pm 2.7$ & $21.0 \pm 4.8$ & $40.9 \pm 3.2$ & $35.7 \pm 3.9$ & $7.8 \pm 0.1$ & $5.6 \pm 2.3$ \\
\hline $6 h$ & $41.3 \pm 3.8$ & $36.5 \pm 1.3$ & $59.2 \pm 4.3$ & $45.7 \pm 4.5$ & $42.4 \pm 2.4$ & $9.1 \pm 3.2$ \\
\hline $6 \mathbf{i}$ & $26.4 \pm 1.3$ & $11.3 \pm 3.5$ & $48.7 \pm 1.2$ & $41.9 \pm 3.7$ & $29.4 \pm 2.5$ & $19.0 \pm 4.1$ \\
\hline $6 \mathbf{j}$ & $30.0 \pm 4.5$ & $20.9 \pm 1.9$ & $44.6 \pm 3.2$ & $38.5 \pm 2.9$ & $31.0 \pm 3.3$ & $30.3 \pm 0.1$ \\
\hline $6 k$ & $37.5 \pm 1.2$ & $26.4 \pm 2.1$ & $40.0 \pm 3.0$ & $25.6 \pm 3.8$ & $31.7 \pm 3.9$ & $28.3 \pm 1.7$ \\
\hline 61 & $30.5 \pm 3.7$ & $15.0 \pm 0.9$ & $47.4 \pm 1.0$ & $34.4 \pm 4.1$ & $41.4 \pm 2.2$ & $21.2 \pm 1.6$ \\
\hline $6 \mathrm{~m}$ & $37.0 \pm 3.9$ & $31.4 \pm 3.1$ & $40.0 \pm 0.7$ & $29.4 \pm 4.0$ & $41.0 \pm 3.2$ & $25.5 \pm 2.2$ \\
\hline $6 n$ & $33.2 \pm 4.1$ & $20.9 \pm 4.2$ & $48.2 \pm 4.2$ & $40.6 \pm 3.2$ & $44.3 \pm 4.8$ & $38.0 \pm 1.0$ \\
\hline 60 & $25.7 \pm 2.0$ & $6.7 \pm 0.1$ & $40.5 \pm 3.2$ & $36.4 \pm 3.0$ & $12.1 \pm 3.7$ & $8.1 \pm 3.3$ \\
\hline $6 p$ & $5.8 \pm 3.9$ & $3.9 \pm 0.4$ & $48.2 \pm 2.9$ & $36.5 \pm 1.7$ & $38.2 \pm 0.8$ & $24.7 \pm 3.6$ \\
\hline $6 q$ & $29.4 \pm 4.0$ & $20.0 \pm 4.7$ & $42.1 \pm 3.0$ & $23.3 \pm 2.6$ & $28.1 \pm 3.5$ & $25.3 \pm 3.5$ \\
\hline $6 r$ & $31.6 \pm 0.7$ & $17.7 \pm 3.3$ & $45.1 \pm 2.3$ & $30.0 \pm 3.3$ & $20.4 \pm 4.7$ & $12.7 \pm 1.1$ \\
\hline $6 s$ & $35.7 \pm 1.9$ & $20.9 \pm 4.5$ & $40.1 \pm 3.1$ & $30.1 \pm 4.2$ & $45.9 \pm 2.1$ & $30.4 \pm 4.5$ \\
\hline $6 t$ & $36.5 \pm 3.2$ & $20.3 \pm 2.0$ & $45.0 \pm 2.1$ & $35.3 \pm 2.7$ & $33.0 \pm 1.9$ & $20.0 \pm 3.3$ \\
\hline $6 u$ & $31.8 \pm 3.1$ & $25.1 \pm 2.4$ & $45.7 \pm 2.6$ & $41.7 \pm 2.2$ & $48.2 \pm 1.7$ & $33.8 \pm 3.3$ \\
\hline $6 v$ & $41.6 \pm 2.8$ & $29.5 \pm 3.5$ & $44.4 \pm 2.5$ & $25.7 \pm 0.4$ & $31.1 \pm 3.9$ & $16.8 \pm 3.0$ \\
\hline $6 w$ & $15.8 \pm 1.2$ & $10.0 \pm 2.0$ & $34.1 \pm 2.5$ & $20.1 \pm 3.5$ & $44.6 \pm 4.5$ & $24.7 \pm 2.8$ \\
\hline $6 x$ & $44.5 \pm 0.5$ & $35.3 \pm 1.6$ & $41.8 \pm 3.1$ & $32.9 \pm 1.8$ & $40.8 \pm 1.8$ & $28.2 \pm 4.2$ \\
\hline $6 y$ & $38.3 \pm 5.0$ & $23.9 \pm 2.7$ & $47.7 \pm 3.1$ & $32.8 \pm 3.0$ & $44.5 \pm 1.4$ & $36.0 \pm 1.7$ \\
\hline $6 z$ & $26.6 \pm 2.8$ & $15.5 \pm 2.4$ & $61.8 \pm 3.6$ & $53.4 \pm 3.0$ & $32.8 \pm 3.6$ & $25.9 \pm 3.2$ \\
\hline 6aa & $39.4 \pm 1.6$ & $25.3 \pm 4.8$ & $43.9 \pm 3.1$ & $35.6 \pm 2.8$ & $41.9 \pm 2.9$ & $18.1 \pm 3.0$ \\
\hline $6 a b$ & $34.2 \pm 2.9$ & $17.5 \pm 4.9$ & $44.8 \pm 2.0$ & $33.8 \pm 2.5$ & $18.4 \pm 1.8$ & $19.0 \pm 0.5$ \\
\hline BT & $58.8 \pm 2.0$ & $41.2 \pm 3.7$ & $56.1 \pm 1.9$ & $46.6 \pm 0.4$ & $52.5 \pm 2.2$ & $46.7 \pm 2.3$ \\
\hline TC & $55.9 \pm 2.4$ & $41.0 \pm 1.4$ & $51.2 \pm 1.1$ & $40.1 \pm 0.5$ & $50.7 \pm 0.4$ & $42.8 \pm 1.9$ \\
\hline
\end{tabular}

${ }^{a}$ Each test was performed in triplicate.

2,4-difluorophenyl) $>\mathbf{6 l}(\mathrm{R}=3$,4-difluorophenyl $)>\mathbf{6 q}$ $(\mathrm{R}=3$,5-difluorophenyl); and $\mathbf{6 g}(\mathrm{R}=2$-chloro-6-methylphenyl $)>6 \mathrm{~b} \quad(\mathrm{R}=3$-chloro-2-methyl-phenyl $)>6 \mathbf{j} \quad(\mathrm{R}=$ 4-chloro-2-methylphenyl); and $6 \mathbf{e}(\mathrm{R}=2$-chloro-4-fluorophenyl) $>\mathbf{6 i}(\mathrm{R}=4$-chloro-3-fluorophenyl); and $\mathbf{6 s}(\mathrm{R}=$ 4-bromo-2-chlorophenyl $)>6 r \quad(\mathrm{R}=4$-bromo-2-methyl- 
phenyl). However, the introduction of nitrogen heterocycles (6z, 6aa and 6ab) did not improve the antibacterial activity against Xoo. Compounds $6 \mathbf{h}(\mathrm{R}=4$-fluoro-3-(trifluoromethyl)phenyl) and $\mathbf{6 z}(\mathrm{R}=5,6$-dichloro-pyridin3-yl) showed slightly higher activity than BT and TC. In terms of controlling Xac, compounds $\mathbf{6 b}(\mathrm{R}=3$-chloro2-methylphenyl), $6 \mathbf{h}$ ( $\mathrm{R}=4$-fluoro-3-(trifluoromethyl)phenyl), 6i ( $\mathrm{R}=4$-chloro-3-fluorophenyl), 6n $(\mathrm{R}=2,4-$ difluorophenyl), $\mathbf{6 u}(\mathrm{R}=2,4,5$-trifluorophenyl) and $\mathbf{6 z}$ ( $\mathrm{R}=5,6$-dichloro-pyridin-3-yl) showed activities of $40.9 \%$, $45.7 \%, 41.9 \%, 40.6 \%, 41.7 \%$, and $53.4 \%$, respectively, at $50 \mathrm{mg} \cdot \mathrm{L}^{-1}$, compared with only $40.1 \%$ for TC. The preliminary SAR study suggested that the activities of halogenated target compounds against $X a c$ were slightly higher than for other substituted target compounds. For example, the antibacterial activities of compounds $6 c \quad(\mathrm{R}=$ 4-methoxy-2-methylphenyl), 6k ( $\mathrm{R}=3$-isopropylphenyl) and $\mathbf{6 w}(\mathrm{R}=3$,4-dimethoxyphenyl) were less than other compounds containing halogen substituents. This may be due to the lipophilicity and stability of halogen atoms. ${ }^{[38-40]}$ Moreover, compound 6e ( $\mathrm{R}=2$-chloro-4-fluorophenyl) showed the highest activity $(58.5 \%)$ against $R$. Solanacearum at $100 \mathrm{mg} \cdot \mathrm{L}^{-1}$ compared with BT $(52.5 \%)$ and TC (50.7\%).

The half maximal effective concentrations $\left(\mathrm{EC}_{50}\right)$ of the most active compounds were further evaluated (Table 2).
The $\mathrm{EC}_{50}$ against $X o o$ of compound $\mathbf{6 d}\left(54 \mathrm{mg} \cdot \mathrm{L}^{-1}\right)$ was slightly lower than BT $\left(59.6 \mathrm{mg} \bullet \mathrm{L}^{-1}\right)$ and TC $(86.3 \mathrm{mg} \bullet$ $\left.\mathrm{L}^{-1}\right)$. The $\mathrm{EC}_{50}$ values against $X a c$ for $\mathbf{6 h}\left(51.2 \mathrm{mg} \bullet \mathrm{L}^{-1}\right)$ and $\mathbf{6 z}\left(60.7 \mathrm{mg} \cdot \mathrm{L}^{-1}\right)$ were much lower than for BT $(76.3$ $\left.\mathrm{mg} \cdot \mathrm{L}^{-1}\right)$ and $\mathbf{T C}\left(101.7 \mathrm{mg} \cdot \mathrm{L}^{-1}\right)$. Compound $\mathbf{6 e}$ also showed a lower $\mathrm{EC}_{50}\left(74.9 \mathrm{mg} \cdot \mathrm{L}^{-1}\right)$ against $R$. solanacearum than TC $\left(79.0 \mathrm{mg} \cdot \mathrm{L}^{-1}\right)$.

\subsection{Insecticidal activity test}

Evaluation of insecticidal activity against $P$. xylostella (Table 3) indicated that some target compounds showed moderate insecticidal activity. For example, mortality rates for both $6 \mathbf{e}(\mathrm{R}=2$-chloro-4-fluorophenyl) and $6 \mathbf{k}(\mathrm{R}=3$ isopropylphenyl) at $500 \mathrm{mg} \cdot \mathrm{L}^{-1}$ were $60 \%$, while $\mathbf{6 b}(\mathrm{R}=$ 3-chloro-2-methylphenyl) and $\mathbf{6 g}(\mathrm{R}=2$-chloro-6-methylphenyl) had a $50 \%$ mortality rate.

\section{Conclusion}

Novel trifluoromethylpyridine amide derivatives were synthesized in this study. Antibacterial bio-assays indicated that these compounds possessed promising efficacy for controlling Xoo, Xac, and $R$. solanacearum. Compound $\mathbf{6 d}$ exhibited the highest activity against Xoo $\left(\mathrm{EC}_{50} 54.1 \mathrm{mg} \bullet\right.$ $\left.\mathrm{L}^{-1}\right)$ and compared favorably with the positive controls BT $\left(59.6 \mathrm{mg} \cdot \mathrm{L}^{-1}\right)$ and $\mathbf{~ T C}\left(86.3 \mathrm{mg} \cdot \mathrm{L}^{-1}\right)$. Compounds $\mathbf{6 h}$ and 6z had significantly lower $\mathrm{EC}_{50}$ values (51.2 and 60.7

Table 2 Results of $\mathrm{EC}_{50}$ values for $\mathbf{6 d}, \mathbf{6 e}, \mathbf{6 h}, \mathbf{6 z}, \mathbf{B T}$, and $\mathbf{T C}^{a}$

\begin{tabular}{|c|c|c|c|c|c|c|}
\hline \multirow{2}{*}{ Compd. } & \multicolumn{2}{|c|}{ Xoo } & \multicolumn{2}{|c|}{$X a c$} & \multicolumn{2}{|c|}{ R. solanacearum } \\
\hline & Regression equation & $\mathrm{EC}_{50} /\left(\mathrm{mg} \bullet \mathrm{L}^{-1}\right)$ & Regression equation & $\mathrm{EC}_{50} /\left(\mathrm{mg} \bullet \mathrm{L}^{-1}\right)$ & Regression equation & $\mathrm{EC}_{50} /\left(\mathrm{mg} \bullet \mathrm{L}^{-1}\right)$ \\
\hline 6d & $y=1.39 x+2.60$ & $54.1 \pm 4.41$ & & & & \\
\hline $6 e$ & & & & & $y=1.15 x+2.81$ & $74.9 \pm 2.75$ \\
\hline $6 h$ & & & $y=1.19 x+3.30$ & $51.2 \pm 6.02$ & & \\
\hline $6 z$ & & & $y=2.68 x+0.25$ & $60.7 \pm 2.84$ & & \\
\hline BT & $y=0.88 x+3.4$ & $59.6 \pm 2.42$ & $y=1.59 x+2.00$ & $76.3 \pm 2.17$ & & \\
\hline TC & $y=0.95 x+3.2$ & $86.3 \pm 7.69$ & $y=1.34 x+2.30$ & $101.7 \pm 1.71$ & $y=1.21 x+2.70$ & $79.0 \pm 3.78$ \\
\hline
\end{tabular}

${ }^{a}$ Each $\mathrm{EC}_{50}$ test was performed in triplicate.

Table 3 Insecticidal activity against $P$. xylostella of target compounds $6 \mathbf{a}$ to $\mathbf{6} \mathbf{a b}^{a}$

\begin{tabular}{cc||cc}
\hline Compd. & Mortality rate $/ \%$ & Compd. & Mortality rate $/ \%$ \\
\hline $\mathbf{6 a}$ & $40 \pm 5$ & $\mathbf{6 p}$ & $20 \pm 5$ \\
$\mathbf{6 b}$ & $50 \pm 0$ & $\mathbf{6 q}$ & $30 \pm 0$ \\
$\mathbf{6 c}$ & $40 \pm 0$ & $\mathbf{6 r}$ & $40 \pm 0$ \\
$\mathbf{6 d}$ & $40 \pm 5$ & $\mathbf{6 s}$ & $40 \pm 0$ \\
$\mathbf{6 e}$ & $60 \pm 0$ & $\mathbf{6 t}$ & $30 \pm 5$ \\
$\mathbf{6 f}$ & $30 \pm 0$ & $\mathbf{6 u}$ & $20 \pm 0$ \\
$\mathbf{6 g}$ & $50 \pm 0$ & $\mathbf{6 v}$ & $20 \pm 0$ \\
$\mathbf{6 h}$ & $30 \pm 3.3$ & $\mathbf{6 w}$ & $20 \pm 0$ \\
$\mathbf{6 i}$ & $20 \pm 0$ & $\mathbf{6 x}$ & $30 \pm 3.3$ \\
$\mathbf{6 j}$ & $20 \pm 0$ & $\mathbf{6 y}$ & $40 \pm 0$ \\
$\mathbf{6 k}$ & $60 \pm 0$ & $\mathbf{6 z}$ & $20 \pm 0$ \\
$\mathbf{6}$ & $30 \pm 0$ & $\mathbf{6 a a}$ & $30 \pm 0$ \\
$\mathbf{6 m}$ & $40 \pm 2.9$ & $\mathbf{6 a b}$ & $30 \pm 0$ \\
$\mathbf{6 n}$ & $40 \pm 0$ & Chlorpyrifos & $100 \pm 0$ \\
$\mathbf{6 o}$ & $20 \pm 0$ & Avermectin & $100 \pm 0$ \\
\hline
\end{tabular}

${ }^{a}$ Each test was performed in triplicate, P. xylostella $500 \mathrm{mg} \cdot \mathrm{L}^{-1}$. $\left.\mathrm{mg} \cdot \mathrm{L}^{-1}\right)$ against Xac than BT $\left(76.3 \mathrm{mg} \cdot \mathrm{L}^{-1}\right)$ and TC $\left(101.7 \mathrm{mg} \cdot \mathrm{L}^{-1}\right)$. Compound $\mathbf{6 e}$ had the highest antibacterial activity $\left(\mathrm{EC}_{50} 74.9 \mathrm{mg} \cdot \mathrm{L}^{-1}\right)$ against $R$. solanacearum, and showed moderate insecticidal activity $(60 \%)$ against $P$. xylostella. This study presents several novel trifluoromethylpyridine amide derivatives as potential lead compounds for the discovery of antibacterial and insecticidal agents.

\section{Experimental}

\subsection{Instruments and reagents}

Reagents were purchased from markets and used directly. Reactions were monitored by thin layer chromatography (TLC). All NMR spectra were acquired on an AVANCE III HD $400 \mathrm{MHz}$ NMR spectrometer (Bruker Corp., Switzerland). Melting points (uncorrected) of target compounds were measured by a XT-4 binocular microscope (Beijing Tech Instrument Co., China). HRMS were performed on a Q Exactive Orbitrap LC-MS (Thermo Sci- 
entific, USA).

\subsection{Chemistry}

4.2.1 General protocol for preparing intermediates 3 and 4

Intermediate 3 was prepared according to our previous work. $^{[34]}$ A mixture of 1 (200.0 mg, $\left.1.0 \mathrm{mmol}\right), \mathrm{K}_{2} \mathrm{CO}_{3}$ (167.9 $\mathrm{mg}, 1.20 \mathrm{mmol})$, and $\mathrm{KI}(85.0 \mathrm{mg}, 506.0 \mu \mathrm{mol})$ in acetonitrile was stirred for $2 \mathrm{~h}$, and then ethyl 2-chloroacetate $(372.0 \mathrm{mg}, 3.0 \mathrm{mmol}$ ) was added. The reaction was monitored by TLC. The reaction mixture was quenched with water and the precipitate was filtered to obtain intermediate 3 which was subsequently hydrolyzed under alkaline conditions to produce intermediate 4 .

\subsubsection{General protocol for synthesis of $\mathbf{6 a} \sim \mathbf{6 a b}$}

According to the reported method, ${ }^{[35]}$ phosphorus oxychloride $(60.0 \mathrm{mg}, 391.0 \mu \mathrm{mol})$ was added to a mixture of intermediate 4 (100.0 mg, $391.3 \mu \mathrm{mol})$, substituted aniline $5(36.4 \mathrm{mg}, 391.3 \mu \mathrm{mol})$, and triethylamine $(39.6 \mathrm{mg}$, $391.3 \mu \mathrm{mol})$ in acetonitrile and the reaction was monitored by TLC. The reaction mixture was poured into water and filtered to obtain target compounds $\mathbf{6 a} \sim \mathbf{6} \mathbf{a b}$ with yields of $63 \% \sim 72 \%$. Physical and chemical data for the target compounds are as follows.

2-((3-Chloro-5-(trifluoromethyl)pyridin-2-yl)oxy)- $\mathrm{N}-(2-$ fluoro-4-methylphenyl)acetamide (6a): Yield 67\%. White solid, m.p. 205 206 ${ }^{\circ} \mathrm{C}$; ${ }^{1} \mathrm{H}$ NMR (400 MHz, DMSO- $d_{6}$ ) $\delta$ : $10.21(\mathrm{~s}, 1 \mathrm{H}), 8.47(\mathrm{~d}, J=1.0 \mathrm{~Hz}, 1 \mathrm{H}), 8.20(\mathrm{~d}, J=2.4$ $\mathrm{Hz}, 1 \mathrm{H}), 7.74$ (t, $J=8.3 \mathrm{~Hz}, 1 \mathrm{H}), 7.20 \sim 7.06$ (m, 1H), 6.97 $(\mathrm{d}, J=8.2 \mathrm{~Hz}, 1 \mathrm{H}), 4.97(\mathrm{~s}, 2 \mathrm{H}), 2.28(\mathrm{~s}, 3 \mathrm{H}) ;{ }^{13} \mathrm{C} \mathrm{NMR}$ (100 MHz, DMSO- $\left.d_{6}\right) \delta: 165.5,158.1,153.7$ (d, $J=244.8$ $\mathrm{Hz}$ ), 140.0 (q, $J=5.3 \mathrm{~Hz}$ ), 136.0 (q, $J=7.3 \mathrm{~Hz}$ ), 134.5, 134.4, 125.3, 125.3, 124.1, 124.1, 123.7 (q, $J=270.0 \mathrm{~Hz}$ ), 123.2 (d, $J=11.8 \mathrm{~Hz}), 116.4(\mathrm{~d}, J=19.1 \mathrm{~Hz}), 107.0$ (q, $J=34.9 \mathrm{~Hz}$ ), 53.3, 20.8; ${ }^{19} \mathrm{~F}$ NMR (376 MHz, DMSO- $d_{6}$ ) $\delta$ : $-60.23,-125.51$. HRMS (ESI) calcd for $\mathrm{C}_{15} \mathrm{H}_{10} \mathrm{O}_{2-}$ $\mathrm{N}_{2} \mathrm{ClF}_{4}[\mathrm{M}-\mathrm{H}]^{-}$361.0361, found 361.0379.

$N$-(3-Chloro-2-methylphenyl)-2-((3-chloro-5-(trifluoromethyl)pyridin-2-yl)oxy)acetamide (6b): Yield 65\%. Pink solid, m.p. 245 246 ${ }^{\circ} \mathrm{C}$; ${ }^{1} \mathrm{H}$ NMR (400 MHz, DMSO- $d_{6}$ ) $\delta: 10.07(\mathrm{~s}, 1 \mathrm{H}), 8.48(\mathrm{~s}, 1 \mathrm{H}), 8.20(\mathrm{~d}, J=2.4 \mathrm{~Hz}, 1 \mathrm{H}), 7.33$ $(\mathrm{s}, 1 \mathrm{H}), 7.31(\mathrm{~s}, 1 \mathrm{H}), 7.21(\mathrm{t}, J=8.0 \mathrm{~Hz}, 1 \mathrm{H}), 4.95(\mathrm{~s}, 2 \mathrm{H})$, $2.26(\mathrm{~s}, 3 \mathrm{H}) ;{ }^{13} \mathrm{C}$ NMR $\left(100 \mathrm{MHz}, \mathrm{DMSO}-d_{6}\right) \delta$ : 165.6, $158.1,140.0$ (q, $J=5.5 \mathrm{~Hz}), 137.6,134.5$ (q, $J=1.9 \mathrm{~Hz})$, $134.3,131.0,127.4,126.9,125.3,124.9,123.7$ (q, $J=$ $270.0 \mathrm{~Hz}), 107.1(\mathrm{q}, J=34.9 \mathrm{~Hz}), 53.4,15.6 ;{ }^{19} \mathrm{~F}$ NMR $\left(376 \mathrm{MHz}, \mathrm{DMSO}-d_{6}\right) \delta$ : -60.21 . HRMS (ESI) calcd for $\mathrm{C}_{15} \mathrm{H}_{10} \mathrm{O}_{2} \mathrm{~N}_{2} \mathrm{Cl}_{2} \mathrm{~F}_{3}[\mathrm{M}-\mathrm{H}]^{-}$377.0066, found 377.0078.

2-((3-Chloro-5-(trifluoromethyl)pyridin-2-yl)oxy)- $N$-(4methoxy-2-methylphenyl)acetamide (6c): Yield 68\%. Pink solid, m.p. $223 \sim 224{ }^{\circ} \mathrm{C}$; ${ }^{1} \mathrm{H}$ NMR (400 MHz, DMSO- $d_{6}$ ) $\delta: 9.67(\mathrm{~s}, 1 \mathrm{H}), 8.47(\mathrm{~d}, J=1.0 \mathrm{~Hz}, 1 \mathrm{H}), 8.18(\mathrm{~d}, J=2.4$ $\mathrm{Hz}, 1 \mathrm{H}), 7.20$ (d, $J=8.7 \mathrm{~Hz}, 1 \mathrm{H}), 6.81(\mathrm{~d}, J=2.7 \mathrm{~Hz}, 1 \mathrm{H})$, 6.73 (dd, $J=8.7,2.9 \mathrm{~Hz}, 1 \mathrm{H}), 4.90$ (s, 2H), 3.72 (s, 3H), $2.19(\mathrm{~s}, 3 \mathrm{H}) ;{ }^{13} \mathrm{C}$ NMR (100 MHz, DMSO-d $) \delta: 165.3$, 158.1, 157.4, 140.1 (q, $J=5.3 \mathrm{~Hz}), 134.4$ (q, $J=4.3 \mathrm{~Hz})$, 128.9, 127.0, 125.3, 123.7 (q, $J=269.7 \mathrm{~Hz}), 115.9,111.7$, $107.0(\mathrm{q}, J=35.0 \mathrm{~Hz}), 55.6,53.3,18.5 ;{ }^{19} \mathrm{~F}$ NMR (376 $\left.\mathrm{MHz}, \mathrm{DMSO}-d_{6}\right) \delta$ : -60.19 . HRMS (ESI) calcd for $\mathrm{C}_{16} \mathrm{H}_{13} \mathrm{O}_{3} \mathrm{~N}_{2} \mathrm{ClF}_{3}[\mathrm{M}-\mathrm{H}]^{-}$373.0561, found 373.0573 .

2-((3-Chloro-5-(trifluoromethyl)pyridin-2-yl)oxy)- $N-(4-$ fluoro-2-methylphenyl)acetamide (6d): Yield 67\%. Pink solid, m.p. 233 $234{ }^{\circ} \mathrm{C} ;{ }^{1} \mathrm{H}$ NMR (400 MHz, DMSO- $d_{6}$ ) $\delta: 9.82(\mathrm{~s}, 1 \mathrm{H}), 8.48(\mathrm{~d}, J=1.0 \mathrm{~Hz}, 1 \mathrm{H}), 8.19(\mathrm{~d}, J=2.4$ $\mathrm{Hz}, 1 \mathrm{H}), 7.35$ (dd, $J=8.8,5.6 \mathrm{~Hz}, 1 \mathrm{H}), 7.11$ (dd, $J=9.7$, $2.8 \mathrm{~Hz}, 1 \mathrm{H}), 7.00(\mathrm{td}, J=8.6,2.9 \mathrm{~Hz}, 1 \mathrm{H}), 4.93(\mathrm{~s}, 2 \mathrm{H})$, $2.23(\mathrm{~s}, 3 \mathrm{H}) ;{ }^{13} \mathrm{C}$ NMR (100 MHz, DMSO-d 6$) \delta: 165.5$, 160.0 (d, $J=241.7 \mathrm{~Hz}), 158.1,140.1$ (q, $J=5.4 \mathrm{~Hz}), 135.4$ (q, $J=8.3 \mathrm{~Hz}), 134.4,132.3,127.4,125.3,123.7$ (q, $J=$ $269.7 \mathrm{~Hz}), 117.2(\mathrm{~d}, J=22.1 \mathrm{~Hz}), 113.1(\mathrm{~d}, J=22.1 \mathrm{~Hz})$, 107.0 (q, $J=35.0 \mathrm{~Hz}), 53.3,18.3 ;{ }^{19} \mathrm{~F}$ NMR $(376 \mathrm{MHz}$, DMSO- $\left.d_{6}\right) \delta$ : $-60.21,-117.35$. HRMS (ESI) calcd for $\mathrm{C}_{15} \mathrm{H}_{10} \mathrm{O}_{2} \mathrm{~N}_{2} \mathrm{ClF}_{4}[\mathrm{M}-\mathrm{H}]^{-}$361.0361, found 361.0375.

$\mathrm{N}$-(2-Chloro-4-fluorophenyl)-2-((3-chloro-5-(trifluoromethyl)pyridin-2-yl)oxy)acetamide (6e): Yield 66\%. White solid, m.p. 236 $237{ }^{\circ} \mathrm{C} ;{ }^{1} \mathrm{H}$ NMR (400 MHz, DMSO-d $)$ $\delta: 10.14(\mathrm{~s}, 1 \mathrm{H}), 8.49(\mathrm{~s}, 1 \mathrm{H}), 8.20(\mathrm{~s}, 1 \mathrm{H}), 7.73 \sim 7.62(\mathrm{~m}$, $1 \mathrm{H}), 7.54(\mathrm{~d}, J=8.5 \mathrm{~Hz}, 1 \mathrm{H}), 7.24(\mathrm{t}, J=8.5 \mathrm{~Hz}, 1 \mathrm{H}), 4.99$ $(\mathrm{s}, 2 \mathrm{H}) ;{ }^{13} \mathrm{C}$ NMR $\left(100 \mathrm{MHz}, \mathrm{DMSO}-d_{6}\right) \delta: 165.9,159.4$ $(\mathrm{d}, J=245.6 \mathrm{~Hz}), 158.1,140.0(\mathrm{q}, J=5.3 \mathrm{~Hz}), 134.5$, $131.42,128.1,128.0(\mathrm{q}, J=5.9 \mathrm{~Hz}), 125.4,123.6$ (q, $J=$ $269.8 \mathrm{~Hz}), 117.2(\mathrm{~d}, J=25.9 \mathrm{~Hz}), 115.1(\mathrm{~d}, J=22.1 \mathrm{~Hz})$, 107.1 (q, $J=35.0 \mathrm{~Hz}), 53.4 ;{ }^{19} \mathrm{~F}$ NMR (376 MHz, DMSO$\left.d_{6}\right) \delta:-60.25,-114.77$. HRMS (ESI) calcd for $\mathrm{C}_{14} \mathrm{H}_{7}-$ $\mathrm{O}_{2} \mathrm{~N}_{2} \mathrm{Cl}_{2} \mathrm{~F}_{4}[\mathrm{M}-\mathrm{H}]^{-}$380.9815, found 380.9829 .

2-((3-Chloro-5-(trifluoromethyl)pyridin-2-yl)oxy)- $\mathrm{N}-(4-$ (trifluoromethoxy)phenyl)acetamide (6f): Yield 63\%. White solid, m.p. 236 $237{ }^{\circ} \mathrm{C}$; ${ }^{1} \mathrm{H}$ NMR (400 MHz, DMSO- $\left.d_{6}\right) \delta: 10.68(\mathrm{~s}, 1 \mathrm{H}), 8.48(\mathrm{~d}, J=1.1 \mathrm{~Hz}, 1 \mathrm{H}), 8.22$ $(\mathrm{d}, J=2.3 \mathrm{~Hz}, 1 \mathrm{H}), 7.68(\mathrm{t}, J=6.1 \mathrm{~Hz}, 2 \mathrm{H}), 7.35$ (d, $J=8.6$ $\mathrm{Hz}, 2 \mathrm{H}), 4.92$ (s, 2H); ${ }^{13} \mathrm{C}$ NMR (100 MHz, DMSO-d $) \delta$ : $165.4,158.1,144.2,144.2,140.0$ (q, $J=5.2 \mathrm{~Hz}$ ), 138.2, $134.5,134.5,126.3$ (q, $J=270.0 \mathrm{~Hz}), 125.3,122.3,120.9$, 120.6 (q, $J=255.8 \mathrm{~Hz}), 107.1(\mathrm{q}, J=35.0 \mathrm{~Hz}), 53.5 ;{ }^{19} \mathrm{~F}$ NMR (376 MHz, DMSO- $\left.d_{6}\right) \delta$ : $-57.08,-60.25$. HRMS (ESI) calcd for $\mathrm{C}_{15} \mathrm{H}_{8} \mathrm{O}_{3} \mathrm{~N}_{2} \mathrm{ClF}_{6}[\mathrm{M}-\mathrm{H}]^{-}$413.0122, found 413.0137.

2-((3-Chloro-5-(trifluoromethyl)pyridin-2-yl)oxy)- $\mathrm{N}-(2-$ chloro-6-methylphenyl)acetamide (6g): Yield 63\%. White solid, m.p. 199 $200{ }^{\circ} \mathrm{C} ;{ }^{1} \mathrm{H}$ NMR (400 MHz, DMSO-d $)$ $\delta: 10.06(\mathrm{~s}, 1 \mathrm{H}), 8.52(\mathrm{~d}, J=1.1 \mathrm{~Hz}, 1 \mathrm{H}), 8.19(\mathrm{~d}, J=2.4$ $\mathrm{Hz}, 1 \mathrm{H}), 7.35$ (dd, $J=7.2,2.2 \mathrm{~Hz}, 1 \mathrm{H}), 7.30 \sim 7.16(\mathrm{~m}$, $2 \mathrm{H}), 4.95(\mathrm{~s}, 2 \mathrm{H}), 2.21(\mathrm{~s}, 3 \mathrm{H}) ;{ }^{13} \mathrm{C} \mathrm{NMR}(100 \mathrm{MHz}$, DMSO- $\left.d_{6}\right) \delta$ : $165.3,158.1,140.0$ (q, $\left.J=5.5 \mathrm{~Hz}\right), 138.9$, 134.4 (q, $J=8.3 \mathrm{~Hz}), 133.4,132.0,129.5,128.6,127.4$, 125.3, 123.7 (q, $J=270.0 \mathrm{~Hz}), 107.0$ (q, $J=35.0 \mathrm{~Hz}), 53.1$, 18.8; ${ }^{19} \mathrm{~F}$ NMR $\left(376 \mathrm{MHz}\right.$, DMSO- $\left.d_{6}\right) \delta$ : -60.19 . HRMS (ESI) calcd for $\mathrm{C}_{15} \mathrm{H}_{10} \mathrm{O}_{2} \mathrm{~N}_{2} \mathrm{Cl}_{2} \mathrm{~F}_{3}[\mathrm{M}-\mathrm{H}]^{-}$377.0066, found 377.0081 .

2-((3-Chloro-5-(trifluoromethyl)pyridin-2-yl)oxy)- $\mathrm{N}-(4-$ fluoro-3-(trifluoromethyl)phenyl)acetamide (6h): Yield $69 \%$. White solid, m.p. 219 220 ${ }^{\circ} \mathrm{C} ;{ }^{1} \mathrm{H}$ NMR (400 MHz, DMSO- $\left.d_{6}\right) \delta: 10.86(\mathrm{~s}, 1 \mathrm{H}), 8.48(\mathrm{~d}, J=1.0 \mathrm{~Hz}, 1 \mathrm{H}), 8.23$ $(\mathrm{d}, J=2.4 \mathrm{~Hz}, 1 \mathrm{H}), 8.10(\mathrm{dd}, J=6.4,2.4 \mathrm{~Hz}, 1 \mathrm{H}), 7.84 \sim$ 
$7.75(\mathrm{~m}, 1 \mathrm{H}), 7.52(\mathrm{t}, J=9.8 \mathrm{~Hz}, 1 \mathrm{H}), 4.93(\mathrm{~s}, 2 \mathrm{H}) ;{ }^{13} \mathrm{C}$ NMR (100 MHz, DMSO- $\left.d_{6}\right) \delta: 165.7,158.1,155.0$ (dd, $J=247.9,2.6 \mathrm{~Hz}), 140.0(\mathrm{dd}, J=10.6,5.2 \mathrm{~Hz}), 135.8$, $134.5,125.5$ (d, $J=8.2 \mathrm{~Hz}), 125.4,123.6$ (q, $J=270.0 \mathrm{~Hz})$, 122. 9 (q, $J=272.1 \mathrm{~Hz}), 118.6,118.4,117.4$ (d, $J=4.8$ $\mathrm{Hz}$ ), 107.2 (q, $J=34.8 \mathrm{~Hz}), 53.5 ;{ }^{19} \mathrm{~F}$ NMR $(376 \mathrm{MHz}$, DMSO- $\left.d_{6}\right) \delta$ : $-60.29,-60.39,-121.96$. HRMS (ESI) calcd for $\mathrm{C}_{15} \mathrm{H}_{7} \mathrm{O}_{2} \mathrm{~N}_{2} \mathrm{ClF}_{7}[\mathrm{M}-\mathrm{H}]^{-}$415.0079, found 415.0091 .

$\mathrm{N}$-(4-chloro-3-fluorophenyl)-2-((3-chloro-5-(trifluoromethyl)pyridin-2-yl)oxy)acetamide (6i): Yield 70\%. White solid, m.p. greater than $250{ }^{\circ} \mathrm{C}$; ${ }^{1} \mathrm{H}$ NMR $(400 \mathrm{MHz}$, DMSO- $\left.d_{6}\right) \delta: 10.82(\mathrm{~s}, 1 \mathrm{H}), 8.48(\mathrm{dd}, J=2.3,1.2 \mathrm{~Hz}, 1 \mathrm{H})$, $8.22(\mathrm{~d}, J=2.5 \mathrm{~Hz}, 1 \mathrm{H}), 7.74(\mathrm{dd}, J=11.8,2.3 \mathrm{~Hz}, 1 \mathrm{H})$, $7.56(\mathrm{t}, J=8.7 \mathrm{~Hz}, 1 \mathrm{H}), 7.39 \sim 7.28(\mathrm{~m}, 1 \mathrm{H}), 4.92(\mathrm{~s}, 2 \mathrm{H})$; ${ }^{13} \mathrm{C}$ NMR (100 MHz, DMSO- $\left.d_{6}\right) \delta: 165.7,158.1,157.5(\mathrm{~d}$, $J=244.4 \mathrm{~Hz}), 140.0$ (q, $J=5.5 \mathrm{~Hz}), 139.4$ (q, $J=8.9 \mathrm{~Hz})$, $134.5,131.3,125.4,123.6$ (q, $J=269.9 \mathrm{~Hz}), 116.4,113.8$ $(\mathrm{d}, J=17.7 \mathrm{~Hz}), 107.7$ (d, $J=25.8 \mathrm{~Hz}), 107.2(\mathrm{q}, J=35.1$ $\mathrm{Hz}$ ), 53.6; ${ }^{19} \mathrm{~F}$ NMR (376 MHz, DMSO- $\left.d_{6}\right) \delta$ : -60.27 , -114.33. HRMS (ESI) calcd for $\mathrm{C}_{14} \mathrm{H}_{7} \mathrm{O}_{2} \mathrm{~N}_{2} \mathrm{Cl}_{2} \mathrm{~F}_{4}[\mathrm{M}-$ $\mathrm{H}]^{-} 380.9815$, found 380.9829 .

$\mathrm{N}$-(4-chloro-2-methylphenyl)-2-((3-chloro-5-(trifluoromethyl)pyridin-2-yl)oxy)acetamide (6j): Yield 70\%. White solid, m.p. 212 $213{ }^{\circ} \mathrm{C}$; ${ }^{1} \mathrm{H}$ NMR (400 MHz, DMSO-d ${ }_{6}$ ) $\delta: 9.85(\mathrm{~s}, 1 \mathrm{H}), 8.48(\mathrm{~d}, J=1.0 \mathrm{~Hz}, 1 \mathrm{H}), 8.20(\mathrm{~d}, J=2.4$ $\mathrm{Hz}, 1 \mathrm{H}), 7.44$ (d, $J=8.6 \mathrm{~Hz}, 1 \mathrm{H}), 7.33$ (d, $J=2.2 \mathrm{~Hz}, 1 \mathrm{H})$, 7.23 (dd, $J=8.6,2.4 \mathrm{~Hz}, 1 \mathrm{H}), 4.95$ (s, 2H), 2.24 (s, 3H); ${ }^{13} \mathrm{C}$ NMR (100 MHz, DMSO- $\left.d_{6}\right) \delta: 165.5,158.1,140.1$ (q, $J=5.3 \mathrm{~Hz}), 135.1,134.4,130.4,129.7,126.7,126.4$, $125.3,123.7$ (q, $J=269.7 \mathrm{~Hz}), 107.0$ (q, $J=35.0 \mathrm{~Hz}), 53.4$, 18.1; ${ }^{19} \mathrm{~F}$ NMR (376 MHz, DMSO- $\left.d_{6}\right) \delta$ : -60.22 . HRMS (ESI) calcd for $\mathrm{C}_{15} \mathrm{H}_{10} \mathrm{O}_{2} \mathrm{~N}_{2} \mathrm{Cl}_{2} \mathrm{~F}_{3}[\mathrm{M}-\mathrm{H}]^{-}$377.0066, found 377.0077 .

2-((3-Chloro-5-(trifluoromethyl)pyridin-2-yl)oxy)- $N$-(3isopropylphenyl)acetamide (6k): Yield $67 \%$. White solid, m.p. $173 \sim 174{ }^{\circ} \mathrm{C} ;{ }^{1} \mathrm{H}$ NMR (400 MHz, DMSO-d 6$) \delta$ : $10.42(\mathrm{~s}, 1 \mathrm{H}), 8.47(\mathrm{~d}, J=1.0 \mathrm{~Hz}, 1 \mathrm{H}), 8.21(\mathrm{~d}, J=2.4 \mathrm{~Hz}$, $1 \mathrm{H}), 7.52$ (s, 1H), 7.35 (d, $J=8.1 \mathrm{~Hz}, 1 \mathrm{H}), 7.23$ (t, $J=7.8$ $\mathrm{Hz}, 1 \mathrm{H}), 6.96$ (d, J=7.6 Hz, 1H), $4.90(\mathrm{~s}, 2 \mathrm{H}), 2.85$ (dt, $J=13.8,6.9 \mathrm{~Hz}, 1 \mathrm{H}), 1.19$ (s, 3H), 1.17 (s, 3H); ${ }^{13} \mathrm{C} \mathrm{NMR}$ $\left(100 \mathrm{MHz}, \mathrm{DMSO}-d_{6}\right) \delta: 165.0,158.1,149.7,140.1$ (q, $J=$ $5.3 \mathrm{~Hz}), 139.0,134.5,134.5,129.3,125.3,123.7$ (q, $J=$ $269.8 \mathrm{~Hz}), 122.3,117.3,117.0,107.0(\mathrm{q}, J=34.9 \mathrm{~Hz})$, 53.5, 33.9, 24.3; ${ }^{19} \mathrm{~F}$ NMR (376 MHz, DMSO- $\left.d_{6}\right) \delta$ : -60.22. HRMS (ESI) calcd for $\mathrm{C}_{17} \mathrm{H}_{15} \mathrm{O}_{2} \mathrm{~N}_{2} \mathrm{ClF}_{3}[\mathrm{M}-$ $\mathrm{H}]^{-}$371.0769, found 371.0781.

2-((3-Chloro-5-(trifluoromethyl)pyridin-2-yl)oxy)- $N$ (3,4-difluorophenyl)acetamide (61): Yield 65\%. White solid, m.p. 221 222 ${ }^{\circ} \mathrm{C}$; ${ }^{1} \mathrm{H}$ NMR (400 MHz, DMSO- $d_{6}$ ) $\delta$ : $10.72(\mathrm{~s}, 1 \mathrm{H}), 8.47(\mathrm{~d}, J=1.0 \mathrm{~Hz}, 1 \mathrm{H}), 8.22(\mathrm{~d}, J=1.6$ $\mathrm{Hz}, 1 \mathrm{H}), 7.74$ (ddd, $J=13.0,7.4,2.5 \mathrm{~Hz}, 1 \mathrm{H}), 7.42$ (dd, $J=19.5,9.2 \mathrm{~Hz}, 1 \mathrm{H}), 7.35 \sim 7.22(\mathrm{~m}, 1 \mathrm{H}), 4.90(\mathrm{~s}, 2 \mathrm{H})$; ${ }^{13} \mathrm{C}$ NMR (100 MHz, DMSO- $\left.d_{6}\right) \delta: 165.4,158.1,149.4$ (dd, $J=243.8,13.1 \mathrm{~Hz}), 145.9(\mathrm{dd}, J=241.8,12.6 \mathrm{~Hz})$, $140.0(\mathrm{q}, J=5.5 \mathrm{~Hz}), 136.0(\mathrm{dd}, J=9.1,2.9 \mathrm{~Hz}), 134.6(\mathrm{q}$, $J=5.3 \mathrm{~Hz}), 125.4,125.0$ (q, $J=269.9 \mathrm{~Hz}), 118.2$ (d, $J=$
$18.1 \mathrm{~Hz}), 115.8(\mathrm{dd}, J=5.9,3.3 \mathrm{~Hz}), 108.6(\mathrm{~d}, J=21.8$ $\mathrm{Hz}$ ), 107.1 (q, $J=35.1 \mathrm{~Hz}), 53.5 ;{ }^{19} \mathrm{~F}$ NMR $(376 \mathrm{MHz}$, DMSO- $\left.d_{6}\right) \quad \delta:-60.27,-136.82,-144.27$. HRMS (ESI) calcd for $\mathrm{C}_{14} \mathrm{H}_{7} \mathrm{O}_{2} \mathrm{~N}_{2} \mathrm{ClF}_{5}[\mathrm{M}-\mathrm{H}]^{-}$365.0111, found 365.0122 .

2-((3-Chloro-5-(trifluoromethyl)pyridin-2-yl)oxy)- $\mathrm{N}$-(4(trifluoromethyl)phenyl)acetamide (6m): Yield 72\%. faint yellow solid, m.p. 236 237 ${ }^{\circ} \mathrm{C}$; ${ }^{1} \mathrm{H}$ NMR $(400 \mathrm{MHz}$, DMSO-d $\left.d_{6}\right) \delta: 10.85(\mathrm{~s}, 1 \mathrm{H}), 8.49(\mathrm{~d}, J=1.1 \mathrm{~Hz}, 1 \mathrm{H}), 8.23$ $(\mathrm{d}, J=2.3 \mathrm{~Hz}, 2 \mathrm{H}), 7.79(\mathrm{~d}, J=8.6 \mathrm{~Hz}, 2 \mathrm{H}), 7.71(\mathrm{~d}, J=$ $8.8 \mathrm{~Hz}, 2 \mathrm{H}) ;{ }^{13} \mathrm{C}$ NMR $\left(100 \mathrm{MHz}, \mathrm{DMSO}-d_{6}\right) \delta: 165.8$, $158.1,142.5,142.5,140.0$ (q, $J=5.4 \mathrm{~Hz}), 134.6,134.5$, 126.7 (q, $J=3.6 \mathrm{~Hz}), 127.5$ (q, $J=271.3 \mathrm{~Hz}), 125.4,124.2$ $(\mathrm{q}, J=32.1 \mathrm{~Hz}), 122.3,119.5,107.1(\mathrm{q}, J=35.0 \mathrm{~Hz}), 53.6$. ${ }^{19} \mathrm{~F}$ NMR (376 MHz, DMSO- $\left.d_{6}\right) \delta$ : $-60.27,-60.40$. HRMS (ESI) calcd for $\mathrm{C}_{15} \mathrm{H}_{8} \mathrm{O}_{2} \mathrm{~N}_{2} \mathrm{ClF}_{6} \quad[\mathrm{M}-\mathrm{H}]$ 397.0173, found 397.0182.

2-((3-Chloro-5-(trifluoromethyl)pyridin-2-yl)oxy)- $N$ (2,4-difluorophenyl)acetamide (6n): Yield 63\%. White solid, m.p. 193 $194{ }^{\circ} \mathrm{C} ;{ }^{1} \mathrm{H}$ NMR (400 MHz, DMSO- $d_{6}$ ) $\delta: 10.33(\mathrm{~s}, 1 \mathrm{H}), 8.48(\mathrm{~d}, J=0.8 \mathrm{~Hz}, 1 \mathrm{H}), 8.20$ (d, $J=2.4$ $\mathrm{Hz}, 1 \mathrm{H}), 7.86(\mathrm{td}, J=9.0,6.2 \mathrm{~Hz}, 1 \mathrm{H}), 7.37$ (ddd, $J=11.5$, 9.1, $2.8 \mathrm{~Hz}, 1 \mathrm{H}), 7.23 \sim 6.98(\mathrm{~m}, 1 \mathrm{H}), 4.97(\mathrm{~s}, 2 \mathrm{H}) ;{ }^{13} \mathrm{C}$ NMR (100 MHz, DMSO- $\left.d_{6}\right) \delta: 165.8,158.1,159.0$ (dd, $J=243.9,11.6 \mathrm{~Hz}), 154.1(\mathrm{dd}, J=248.5,12.5 \mathrm{~Hz}), 140.0$ (q, $J=5.4 \mathrm{~Hz}), 134.5,125.6(\mathrm{dd}, J=9.4,2.9 \mathrm{~Hz}), 125.4$, $123.6(\mathrm{q}, J=270.0 \mathrm{~Hz}), 122.6(\mathrm{dd}, J=11.9,3.7 \mathrm{~Hz}), 111.7$ (dd, $J=22.0,3.6 \mathrm{~Hz}), 107.1$ (q, $J=35.0 \mathrm{~Hz}$ ), 104.8 (dd, $J=26.8,24.0 \mathrm{~Hz}), 53.3 ;{ }^{19} \mathrm{~F}$ NMR $\left(376 \mathrm{MHz}, \mathrm{DMSO}-d_{6}\right)$ $\delta$ : $-60.25,-114.45,-119.87$. HRMS (ESI) calcd for $\mathrm{C}_{14} \mathrm{H}_{7} \mathrm{O}_{2} \mathrm{~N}_{2} \mathrm{ClF}_{5}[\mathrm{M}-\mathrm{H}]^{-}$365.0111, found 365.0120.

2-((3-Chloro-5-(trifluoromethyl)pyridin-2-yl)oxy)- $N$-(2fluoro-5-methylphenyl)acetamide (6o): Yield 69\%. White solid, m.p. 212 213 ${ }^{\circ} \mathrm{C} ;{ }^{1} \mathrm{H}$ NMR (400 MHz, DMSO- $d_{6}$ ) $\delta: 10.26(\mathrm{~s}, 1 \mathrm{H}), 8.47(\mathrm{~s}, 1 \mathrm{H}), 8.20(\mathrm{~d}, J=2.4 \mathrm{~Hz}, 1 \mathrm{H}), 7.74$ $(\mathrm{d}, J=6.3 \mathrm{~Hz}, 1 \mathrm{H}), 7.16(\mathrm{dd}, J=10.9,8.4 \mathrm{~Hz}, 1 \mathrm{H}), 6.96$ (dd, $J=6.6,4.3 \mathrm{~Hz}, 1 \mathrm{H}), 4.98(\mathrm{~s}, 2 \mathrm{H}), 2.25$ (s, 3H); ${ }^{13} \mathrm{C}$ NMR (100 MHz, DMSO- $\left.d_{6}\right) \delta: 165.7,158.1,151.9(\mathrm{~d}, J=$ $242.4 \mathrm{~Hz}$ ), 140.0 (q, $J=5.2 \mathrm{~Hz}), 134.5,134.2$ (d, $J=43.4$ $\mathrm{Hz}), 126.1,125.6,125.3,123.6$ (q, $J=269.7 \mathrm{~Hz}$ ), 124.4, $115.6(\mathrm{~d}, J=19.2 \mathrm{~Hz}), 107.1$ (q, $J=35.0 \mathrm{~Hz}), 53.4,20.9$. ${ }^{19} \mathrm{~F}$ NMR (376 MHz, DMSO- $\left.d_{6}\right) \delta$ : $-60.25,-130.01$. HRMS (ESI) calcd for $\mathrm{C}_{15} \mathrm{H}_{10} \mathrm{O}_{2} \mathrm{~N}_{2} \mathrm{ClF}_{4}[\mathrm{M}-\mathrm{H}]$ 361.0361 , found 361.0374 .

2-((3-Chloro-5-(trifluoromethyl)pyridin-2-yl)oxy)- $\mathrm{N}-(5$ fluoro-2-methylphenyl)acetamide (6p): Yield 66\%. White solid, m.p. 228 $229{ }^{\circ} \mathrm{C}$; ${ }^{1} \mathrm{H}$ NMR (400 MHz, DMSO- $d_{6}$ ) $\delta$ : $9.85(\mathrm{~s}, 1 \mathrm{H}), 8.48(\mathrm{~s}, 1 \mathrm{H}), 8.20(\mathrm{~d}, J=2.3 \mathrm{~Hz}, 1 \mathrm{H}), 7.39$ $(\mathrm{dd}, J=10.9,2.5 \mathrm{~Hz}, 1 \mathrm{H}), 7.29 \sim 7.23(\mathrm{~m}, 1 \mathrm{H}), 6.98 \sim 6.90$ (m, 1H), 4.98 (s, 2H), $2.24(\mathrm{~s}, 3 \mathrm{H}) ;{ }^{13} \mathrm{C}$ NMR (100 MHz, DMSO- $\left.d_{6}\right) \delta: 165.7,160.5(\mathrm{~d}, J=240.1 \mathrm{~Hz}), 158.1,140.1$ $(\mathrm{q}, J=5.2 \mathrm{~Hz}), 137.4(\mathrm{~d}, J=10.6 \mathrm{~Hz}), 134.4,132.0(\mathrm{~d}, J=$ $9.0 \mathrm{~Hz}), 126.9,125.3,123.7$ (q, $J=269.9 \mathrm{~Hz}), 112.1$ (d, $J=20.7 \mathrm{~Hz}), 111.0(\mathrm{~d}, J=24.8 \mathrm{~Hz}), 107.2$ (q, $J=35.0 \mathrm{~Hz})$, 53.4, 17.6; ${ }^{19} \mathrm{~F}$ NMR (376 MHz, DMSO- $\left.d_{6}\right) \delta$ : -60.23 , -116.55. HRMS (ESI) calcd for $\mathrm{C}_{15} \mathrm{H}_{10} \mathrm{O}_{2} \mathrm{~N}_{2} \mathrm{ClF}_{4}[\mathrm{M}-$ $\mathrm{H}]^{-}$361.0361, found 361.0374 . 
2-((3-Chloro-5-(trifluoromethyl)pyridin-2-yl)oxy)- $N$ (3,5-difluorophenyl)acetamide (6q): Yield 67\%. White solid, m.p. 243 $244{ }^{\circ} \mathrm{C}$; ${ }^{1} \mathrm{H}$ NMR (400 MHz, DMSO-d $\left.d_{6}\right)$ $\delta: 10.89(\mathrm{~s}, 1 \mathrm{H}), 8.48(\mathrm{dd}, J=2.3,1.1 \mathrm{~Hz}, 1 \mathrm{H}), 8.23(\mathrm{~d}, J=$ $2.3 \mathrm{~Hz}, 1 \mathrm{H}), 7.35 \sim 7.22(\mathrm{~m}, 2 \mathrm{H}), 6.97(\mathrm{t}, J=9.3 \mathrm{~Hz}, 1 \mathrm{H})$, $4.92(\mathrm{~s}, 2 \mathrm{H}) ;{ }^{13} \mathrm{C}$ NMR (100 MHz, DMSO- $\left.d_{6}\right) \delta$ : 165.9, $163.0(\mathrm{dd}, J=243.5,15.3 \mathrm{~Hz}), 158.1,141.4(\mathrm{q}, J=13.7$ $\mathrm{Hz}), 140.0$ (dd, $J=10.6,5.2 \mathrm{~Hz}), 134.6,125.3,123.6$ (q, $J=269.9 \mathrm{~Hz}), 107.2(\mathrm{q}, J=35.0 \mathrm{~Hz}), 102.5$ (d, $J=29.4$ $\mathrm{Hz}), 99.4$ (d, $J=25.8 \mathrm{~Hz}), 53.6 ;{ }^{19} \mathrm{~F}$ NMR $(376 \mathrm{MHz}$, DMSO- $\left.d_{6}\right) \delta$ : $-60.29,-108.82,-108.85$. HRMS (ESI) calcd for $\mathrm{C}_{14} \mathrm{H}_{7} \mathrm{O}_{2} \mathrm{~N}_{2} \mathrm{ClF}_{5}[\mathrm{M}-\mathrm{H}]^{-}$365.0111, found 365.0119 .

$\mathrm{N}$-(4-Bromo-2-methylphenyl)-2-((3-chloro-5-(trifluoromethyl)pyridin-2-yl)oxy)acetamide (6r): Yield 64\%. White solid, m.p. $>250{ }^{\circ} \mathrm{C} ;{ }^{1} \mathrm{H}$ NMR (400 MHz, DMSO- $\left.d_{6}\right) \delta$ : 9.85 (s, 1H), 8.47 (d, $J=1.0 \mathrm{~Hz}, 1 \mathrm{H}), 8.20(\mathrm{~d}, J=2.4 \mathrm{~Hz}$, $1 \mathrm{H}), 7.47(\mathrm{~d}, J=1.8 \mathrm{~Hz}, 1 \mathrm{H}), 7.43 \sim 7.31(\mathrm{~m}, 2 \mathrm{H}), 4.94(\mathrm{~s}$, $2 \mathrm{H}), 2.24(\mathrm{~s}, 3 \mathrm{H}) ;{ }^{13} \mathrm{C}$ NMR $\left(100 \mathrm{MHz}, \mathrm{DMSO}-d_{6}\right) \delta$ : $165.5,158.1,140.1$ (q, $J=5.3 \mathrm{~Hz}), 135.5,134.7,134.5$, 133.3, 129.3, 127.0, 125.3, 123.7 (q, $J=269.8 \mathrm{~Hz}$ ), 118.0, $107.0(\mathrm{q}, J=35.0 \mathrm{~Hz}), 53.4,18.0 ;{ }^{19} \mathrm{~F}$ NMR $(376 \mathrm{MHz}$, DMSO- $\left.d_{6}\right) \delta$ : -60.21 . HRMS (ESI) calcd for $\mathrm{C}_{15} \mathrm{H}_{10} \mathrm{O}_{2-}$ $\mathrm{N}_{2} \mathrm{BrClF}_{3}[\mathrm{M}-\mathrm{H}]^{-}$420.9561, found 420.9573 .

$\mathrm{N}$-(4-Bromo-2-chlorophenyl)-2-((3-chloro-5-(trifluoromethyl)pyridin-2-yl)oxy)acetamide (6s): Yield 70\%. White solid, m.p. 216 217 ${ }^{\circ} \mathrm{C}$; ${ }^{1} \mathrm{H}$ NMR (400 MHz, DMSO- $d_{6}$ ) $\delta$ : $10.18(\mathrm{~s}, 1 \mathrm{H}), 8.49(\mathrm{~d}, J=1.1 \mathrm{~Hz}, 1 \mathrm{H}), 8.21(\mathrm{~d}, J=2.5$ $\mathrm{Hz}, 1 \mathrm{H}), 7.81$ (d, $J=2.2 \mathrm{~Hz}, 1 \mathrm{H}), 7.72(\mathrm{~d}, J=8.8 \mathrm{~Hz}, 1 \mathrm{H})$, 7.55 (dd, $J=8.7,2.2 \mathrm{~Hz}, 1 \mathrm{H}), 5.01(\mathrm{~s}, 2 \mathrm{H}) ;{ }^{13} \mathrm{C}$ NMR $(100$ $\left.\mathrm{MHz}, \mathrm{DMSO}-d_{6}\right) \delta$ : $166.0,158.1,144.8,140.0$ (q, $J=5.3$ $\mathrm{Hz}), 134.3$, 132.2, 131.2, 131.0, 127.4, 125.4, 123.6 (q, $J=$ $270.0 \mathrm{~Hz}$ ), 117.2, 107.1 (q, $J=35.0 \mathrm{~Hz}), 53.4 ;{ }^{19} \mathrm{~F}$ NMR (376 MHz, DMSO-d $) \delta$ : -60.25 . HRMS (ESI) calcd for $\mathrm{C}_{14} \mathrm{H}_{7} \mathrm{O}_{2} \mathrm{~N}_{2} \mathrm{BrCl}_{2} \mathrm{~F}_{3}[\mathrm{M}-\mathrm{H}]^{-} \quad 440.9015$, found 440.9021

2-((3-Chloro-5-(trifluoromethyl)pyridin-2-yl)oxy)- $N$-(3methoxy-5-(trifluoromethyl)phenyl)acetamide (6t): Yield $67 \%$. Faint yellow solid, m.p. 243 244 ${ }^{\circ} \mathrm{C} ;{ }^{1} \mathrm{H}$ NMR (400 $\left.\mathrm{MHz}, \mathrm{DMSO}-d_{6}\right) \delta: 10.82(\mathrm{~s}, 1 \mathrm{H}), 8.48(\mathrm{~d}, J=1.1 \mathrm{~Hz}, 1 \mathrm{H})$, $8.22(\mathrm{~d}, J=2.5 \mathrm{~Hz}, 1 \mathrm{H}), 7.59(\mathrm{~s}, 1 \mathrm{H}), 7.42(\mathrm{~s}, 1 \mathrm{H}), 6.98$ (s, $1 \mathrm{H}), 4.93(\mathrm{~s}, 2 \mathrm{H}), 3.82(\mathrm{~s}, 3 \mathrm{H}) ;{ }^{13} \mathrm{C} \mathrm{NMR}(100 \mathrm{MHz}$, DMSO- $\left.d_{6}\right) \delta$ : 166.0, 158.1, 144.8, 140.0 (q, $J=5.3 \mathrm{~Hz}$ ), $134.3,123.6$ (q, $J=270.0 \mathrm{~Hz}), 131.2,131.0,130.9,127.4$, $125.4,117.8,117.2,107.1(\mathrm{q}, J=35.0 \mathrm{~Hz}), 106.2,53.4 .{ }^{19} \mathrm{~F}$ NMR (376 MHz, DMSO- $\left.d_{6}\right) \delta$ : -60.25 . HRMS (ESI) calcd for $\mathrm{C}_{16} \mathrm{H}_{10} \mathrm{O}_{3} \mathrm{~N}_{2} \mathrm{ClF}_{6}[\mathrm{M}-\mathrm{H}]^{-}$427.0279, found 427.0289 .

2-((3-Chloro-5-(trifluoromethyl)pyridin-2-yl)oxy)- $N$ (2,4,5-trifluorophenyl)acetamide (6u): Yield 66\%. White solid, m.p. 217 $218{ }^{\circ} \mathrm{C}$; ${ }^{1} \mathrm{H}$ NMR (400 MHz, DMSO-d $d_{6}$ ) $\delta$ : $10.55(\mathrm{~s}, 1 \mathrm{H}), 8.47(\mathrm{~d}, J=1.1 \mathrm{~Hz}, 1 \mathrm{H}), 8.20(\mathrm{t}, J=5.2$ $\mathrm{Hz}, 1 \mathrm{H}), 8.12 \sim 7.94(\mathrm{~m}, 1 \mathrm{H}), 7.70(\mathrm{td}, J=10.7,7.5 \mathrm{~Hz}$, 1H), $4.99(\mathrm{~s}, 2 \mathrm{H}) ;{ }^{13} \mathrm{C}$ NMR (100 MHz, DMSO-d $) \delta$ : $166.1,158.1,148.9$ (dd, $J=247.0,9.5 \mathrm{~Hz}), 145.6$ (dd, $J=$ $241.7,33.6 \mathrm{~Hz}), 140.0$ (q, $J=5.5 \mathrm{~Hz}), 134.5,134.5,125.4$, 125.0 (q, $J=269.9 \mathrm{~Hz}), 122.9(\mathrm{dd}, J=13.5,9.4 \mathrm{~Hz}), 111.7$ $(\mathrm{dd}, J=23.7,2.6 \mathrm{~Hz}), 107.2$ (q, $J=34.9 \mathrm{~Hz}), 106.5$ (dd,
$J=25.9,22.2 \mathrm{~Hz}), 53.4 ;{ }^{19} \mathrm{~F}$ NMR $\left(376 \mathrm{MHz}, \mathrm{DMSO}-d_{6}\right)$ $\delta$ : $-60.29,-126.05,-139.58,-141.59$. HRMS (ESI) calcd for $\mathrm{C}_{14} \mathrm{H}_{6} \mathrm{O}_{2} \mathrm{~N}_{2} \mathrm{ClF}_{6}[\mathrm{M}-\mathrm{H}]^{-}$383.0017, found 383.0030 .

2-((3-Chloro-5-(trifluoromethyl)pyridin-2-yl)oxy)- $\mathrm{N}-(4-$ (difluoromethoxy)phenyl)acetamide (6v): Yield 71\%. Yellow solid, m.p. 234 235 ${ }^{\circ} \mathrm{C}$; ${ }^{1} \mathrm{H}$ NMR $(400 \mathrm{MHz}$, DMSO- $\left.d_{6}\right) \delta: 10.55(\mathrm{~s}, 1 \mathrm{H}), 8.47(\mathrm{~s}, 1 \mathrm{H}), 8.21(\mathrm{~s}, 1 \mathrm{H})$, $7.79 \sim 7.52(\mathrm{~m}, 2 \mathrm{H}), 7.52 \sim 6.92(\mathrm{~m}, 3 \mathrm{H}), 4.90(\mathrm{~s}, 2 \mathrm{H}) ;{ }^{13} \mathrm{C}$ NMR (100 MHz, DMSO- $\left.d_{6}\right) \delta: 165.1,158.1,147.0$ (q, $J=$ $3.2 \mathrm{~Hz}$ ), 140.1 (q, $J=5.4 \mathrm{~Hz}), 136.2,134.5,134.5,125.3$, 123.7 (q, $J=269.8 \mathrm{~Hz}), 120.9,120.1,116.9$ (q, $J=257.7$ $\mathrm{Hz}$ ), 107.1 (q, $J=35.0 \mathrm{~Hz}$ ), 53.5; ${ }^{19} \mathrm{~F}$ NMR (376 MHz, DMSO- $\left.d_{6}\right) \delta$ : $-60.23,-81.55,-81.74$. HRMS (ESI) calcd for $\mathrm{C}_{15} \mathrm{H}_{9} \mathrm{O}_{3} \mathrm{~N}_{2} \mathrm{ClF}_{5}[\mathrm{M}-\mathrm{H}]^{-}$395.0216, found 395.0228 .

2-((3-Chloro-5-(trifluoromethyl)pyridin-2-yl)oxy)- $\mathrm{N}-(3$, 4-dimethoxyphenyl)acetamide (6w): Yield 69\%. Brown solid, m.p. 204 205 ${ }^{\circ} \mathrm{C} ;{ }^{1} \mathrm{H}$ NMR (400 MHz, DMSO-d $)$ $\delta: 10.35(\mathrm{~s}, 1 \mathrm{H}), 8.62 \sim 8.38(\mathrm{~m}, 1 \mathrm{H}), 8.22(\mathrm{~d}, J=2.5 \mathrm{~Hz}$, $1 \mathrm{H}), 7.34(\mathrm{~d}, J=2.4 \mathrm{~Hz}, 1 \mathrm{H}), 7.03(\mathrm{dd}, J=8.7,2.4 \mathrm{~Hz}$, $1 \mathrm{H}), 6.91(\mathrm{~d}, J=8.8 \mathrm{~Hz}, 1 \mathrm{H}), 4.87(\mathrm{~s}, 2 \mathrm{H}), 3.72$ (d, $J=1.5$ $\mathrm{Hz}, 6 \mathrm{H}) ;{ }^{13} \mathrm{C}$ NMR $\left(100 \mathrm{MHz}, \mathrm{DMSO}-d_{6}\right) \delta$ : 164.6, 158.1, $149.0,145.4,140.1$ (q, $J=5.2 \mathrm{~Hz}), 134.5,132.6,125.3$, $125.0(\mathrm{q}, J=269.9 \mathrm{~Hz}), 112.5,111.2,107.0(\mathrm{q}, J=35.0$ $\mathrm{Hz}), 104.4,56.1,55.7,53.5 ;{ }^{19} \mathrm{~F}$ NMR (376 MHz, DMSO$\left.d_{6}\right) \delta$ : -60.21 . HRMS (ESI) calcd for $\mathrm{C}_{16} \mathrm{H}_{13} \mathrm{O}_{4} \mathrm{~N}_{2} \mathrm{ClF}_{3}$ $[\mathrm{M}-\mathrm{H}]^{-}$389.0511, found 389.0525.

$\mathrm{N}$-(4-Bromo-3-fluorophenyl)-2-((3-chloro-5-(trifluoromethyl)pyridin-2-yl)oxy)acetamide (6x): Yield $70 \%$. White solid, m.p. $>250{ }^{\circ} \mathrm{C} ;{ }^{1} \mathrm{H}$ NMR (400 MHz, DMSO- $\left.d_{6}\right) \delta: 10.83(\mathrm{~s}, 1 \mathrm{H}), 8.48(\mathrm{dd}, J=2.3,1.2 \mathrm{~Hz}, 1 \mathrm{H})$, $8.23(\mathrm{~d}, J=2.5 \mathrm{~Hz}, 1 \mathrm{H}), 7.75 \sim 7.62(\mathrm{~m}, 2 \mathrm{H}), 7.32 \sim 7.20$ $(\mathrm{m}, 1 \mathrm{H}), 4.91(\mathrm{~s}, 2 \mathrm{H}) ;{ }^{13} \mathrm{C}$ NMR (100 MHz, DMSO- $\left.d_{6}\right) \delta$ : $165.7,158.5$ (d, $J=242.7 \mathrm{~Hz}), 158.1,140.0$ (q, $J=7.9 \mathrm{~Hz})$, 134.6, 134.1, 125.4, 123.6 (q, $J=269.6 \mathrm{~Hz}), 116.9,116.9$, 107.6 (d, $J=27.2 \mathrm{~Hz}), 106.8$ (q, $J=34.9 \mathrm{~Hz}), 101.7$ (d, $J=21.0 \mathrm{~Hz}), 53.6 ;{ }^{19} \mathrm{~F}$ NMR $\left(376 \mathrm{MHz}, \mathrm{DMSO}-d_{6}\right) \delta$ : $-60.26,-106.42$. HRMS (ESI) calcd for $\mathrm{C}_{14} \mathrm{H}_{7} \mathrm{O}_{2} \mathrm{~N}_{2} \mathrm{Br}-$ $\mathrm{ClF}_{4}[\mathrm{M}-\mathrm{H}]^{-}$424.9310, found 424.9321.

2-((3-Chloro-5-(trifluoromethyl)pyridin-2-yl)oxy)- $N$ (2,6-difluorophenyl)acetamide (6y): Yield 67\%. White solid, m.p. 208 $209{ }^{\circ} \mathrm{C}$; ${ }^{1} \mathrm{H}$ NMR (400 MHz, DMSO-d $)$ $\delta: 10.26(\mathrm{~s}, 1 \mathrm{H}), 8.51(\mathrm{dd}, J=2.3,1.1 \mathrm{~Hz}, 1 \mathrm{H}), 8.20(\mathrm{~d}, J=$ $2.4 \mathrm{~Hz}, 1 \mathrm{H}), 7.37(\mathrm{dq}, J=8.0,6.3 \mathrm{~Hz}, 1 \mathrm{H}), 7.26 \sim 7.11(\mathrm{~m}$, $2 \mathrm{H}), 4.98(\mathrm{~s}, 2 \mathrm{H}) ;{ }^{13} \mathrm{C} \mathrm{NMR}\left(100 \mathrm{MHz}, \mathrm{DMSO}-d_{6}\right) \delta$ : $165.6,158.0,157.9$ (dd, $J=249.2,5.0 \mathrm{~Hz}), 140.0$ (q, $J=$ $5.3 \mathrm{~Hz}), 134.5,134.5,128.7 \quad 125.4,123.6$ (q, $J=269.8$ $\mathrm{Hz}), 114.2$ (d, $J=17.0 \mathrm{~Hz}), 112.4(\mathrm{dd}, J=18.2,4.9 \mathrm{~Hz})$, $107.1(\mathrm{q}, J=34.9 \mathrm{~Hz}), 52.9 ;{ }^{19} \mathrm{~F}$ NMR $(376 \mathrm{MHz}$, DMSO- $\left.d_{6}\right) \delta:-60.26,-117.53,-117.55$. HRMS (ESI) calcd for $\mathrm{C}_{14} \mathrm{H}_{7} \mathrm{O}_{2} \mathrm{~N}_{2} \mathrm{ClF}_{5}[\mathrm{M}-\mathrm{H}]^{-}$365.0111, found 365.0122 .

2-((3-Chloro-5-(trifluoromethyl)pyridin-2-yl)oxy)- $N$ (5,6-dichloropyridin-3-yl)acetamide (6z): Yield 66\%. Faint yellow solid, m.p. $218 \sim 219{ }^{\circ} \mathrm{C}$; ${ }^{1} \mathrm{H}$ NMR $(400 \mathrm{MHz}$, DMSO- $\left.d_{6}\right) \delta: 11.08(\mathrm{~s}, 1 \mathrm{H}), 8.52(\mathrm{~d}, J=2.4 \mathrm{~Hz}, 1 \mathrm{H}), 8.49$ 
$(\mathrm{d}, J=1.1 \mathrm{~Hz}, 1 \mathrm{H}), 8.36(\mathrm{~d}, J=2.4 \mathrm{~Hz}, 1 \mathrm{H}), 8.24(\mathrm{~d}, J=$ $2.4 \mathrm{~Hz}, 1 \mathrm{H}), 4.96(\mathrm{~s}, 2 \mathrm{H}) ;{ }^{13} \mathrm{C}$ NMR (100 MHz, DMSO-d $)$ $\delta: 166.3,158.1,141.5,139.9(\mathrm{q}, J=5.1 \mathrm{~Hz}), 138.8,136.1$, 134.6, 129.3, 129.2, 125.4, 123.6 (q, $J=269.8 \mathrm{~Hz}$ ), 107.2 $(\mathrm{q}, J=35.0 \mathrm{~Hz}), 53.5 ;{ }^{19} \mathrm{~F}$ NMR (376 MHz, DMSO- $\left.d_{6}\right) \delta$ : -60.30. HRMS (ESI) calcd for $\mathrm{C}_{13} \mathrm{H}_{6} \mathrm{O}_{2} \mathrm{~N}_{3} \mathrm{Cl}_{3} \mathrm{~F}_{3}[\mathrm{M}-$ $\mathrm{H}]^{-}$397.9472, found 397.9481.

2-((3-Chloro-5-(trifluoromethyl)pyridin-2-yl)oxy)- $N-(2$, 6-dichloropyridin-3-yl)acetamide (6aa): Yield 65\%. Pink solid, m.p. 191 $192{ }^{\circ} \mathrm{C}$; ${ }^{1} \mathrm{H}$ NMR (400 MHz, DMSO- $d_{6}$ ) $\delta: 10.41(\mathrm{~s}, 1 \mathrm{H}), 8.63 \sim 8.41(\mathrm{~m}, 1 \mathrm{H}), 8.28(\mathrm{~d}, J=8.5 \mathrm{~Hz}$, $1 \mathrm{H}), 8.22$ (d, $J=2.4 \mathrm{~Hz}, 1 \mathrm{H}), 7.59$ (d, $J=8.5 \mathrm{~Hz}, 1 \mathrm{H}), 5.05$ $(\mathrm{s}, 2 \mathrm{H}) ;{ }^{13} \mathrm{C}$ NMR $\left(100 \mathrm{MHz}, \mathrm{DMSO}-d_{6}\right) \delta: 166.6,158.1$, $144.1,141.5,140.0$ (q, $J=5.2 \mathrm{~Hz}$ ), 136.8, 134.6, 131.6, 125.4, 124.4, 123.6 (q, $J=269.8 \mathrm{~Hz}), 107.2$ (q, $J=35.0$ $\mathrm{Hz}$ ), 53.4; ${ }^{19} \mathrm{~F}$ NMR (376 MHz, DMSO- $\left.d_{6}\right) \delta$ : -60.28 . HRMS (ESI) calcd for $\mathrm{C}_{13} \mathrm{H}_{6} \mathrm{O}_{2} \mathrm{~N}_{3} \mathrm{Cl}_{3} \mathrm{~F}_{3} \quad[\mathrm{M}-\mathrm{H}]$ 397.9472, found 397.9481.

2-((3-Chloro-5-(trifluoromethyl)pyridin-2-yl)oxy)- $N$ (6-(trifluoromethyl)pyridin-3-yl)acetamide (6ab): Yield $71 \%$. Faint yellow solid, m.p. 197 $198{ }^{\circ} \mathrm{C} ;{ }^{1} \mathrm{H}$ NMR (400 MHz, DMSO- $\left.d_{6}\right) \delta: 11.14(\mathrm{~s}, 1 \mathrm{H}), 8.89(\mathrm{~d}, J=2.4 \mathrm{~Hz}, 1 \mathrm{H})$, $8.53(\mathrm{dd}, J=8.0,6.8 \mathrm{~Hz}, 1 \mathrm{H}), 8.38$ (d, $J=2.4 \mathrm{~Hz}, 1 \mathrm{H})$, $8.29(\mathrm{dd}, J=8.6,2.3 \mathrm{~Hz}, 1 \mathrm{H}), 7.91$ (d, $J=8.6 \mathrm{~Hz}, 1 \mathrm{H})$, $4.98(\mathrm{~s}, 2 \mathrm{H}) ;{ }^{13} \mathrm{C}$ NMR (100 MHz, DMSO-d $) \delta: 166.4$, $158.3,141.5$ (q, $J=34.8 \mathrm{~Hz}), 141.0,140.7$ (q, $J=5.3 \mathrm{~Hz})$, $138.6,138.2,127.1,124.8$ (q, $J=270.0 \mathrm{~Hz}), 122.2$ (q, $J=$ $273.2 \mathrm{~Hz}), 121.9,120.8,107.9$ (q, $J=34.9 \mathrm{~Hz}), 53.8 ;{ }^{19} \mathrm{~F}$ NMR $\left(376 \mathrm{MHz}, \mathrm{DMSO}-d_{6}\right) \delta$ : $-60.21,-65.75$. HRMS (ESI) calcd for $\mathrm{C}_{14} \mathrm{H}_{7} \mathrm{O}_{2} \mathrm{~N}_{3} \mathrm{ClF}_{6}[\mathrm{M}-\mathrm{H}]^{-}$398.0126, found 398.0139 .

\subsection{Evaluation of antibacterial activity in vitro}

In vitro antibacterial activity of compounds $\mathbf{6 a} \sim \mathbf{6} \mathbf{a b}$ against Xoo, Xac, and $R$. solanacearum, was evaluated as follows. ${ }^{[36-37]}$ Solutions $(1 \mathrm{~mL})$ of the synthesized compounds BT or TC at various concentrations were added to $4 \mathrm{~mL}$ of nutrient broth (NB) medium. Suspensions of Xoo, $X a c$, or $R$. solanacearum bacteria $(40 \mu \mathrm{L})$ were then added and the inoculated samples cultured on a shaker at 180 $\mathrm{r} / \mathrm{min}$ and $(28 \pm 1){ }^{\circ} \mathrm{C}$; The negative control reached the logarithmic growth phase after $24 \sim 48 \mathrm{~h}$. The optical density at $595 \mathrm{~nm}$ (OD 595) for each culture was determined by Model 680 microplate reader (Bio-Rad, Hercules, CA). The inhibition rate $(Y)$ was calculated as follows:

Inhibition rate: $Y / \%=(C-T) / C \times 100$

where $C$ is the corrected OD 595 of untreated NB media and $T$ is the corrected OD 595 of treated NB media.

\subsection{Insecticidal activity against $P$. xylostella}

The insecticidal bioassay was carried out at $(25 \pm$ 1) ${ }^{\circ} \mathrm{C} .{ }^{[41]}$ Mortality rates were calculated using Abbott's formula. ${ }^{[42]}$ Following the reported method, ${ }^{[43]}$ discs of fresh cabbage ( $2 \mathrm{~cm}$ diameter) were immersed in a 500 $\mathrm{mg} \bullet \mathrm{L}^{-1}$ solution of a target compound or a positive control insecticide (chlorpyrifos or avermectin). Distilled water was used as a blank control. The discs were then placed in a $9 \mathrm{~cm}$ diameter Petri dish with wet filter paper to retain moisture. Twenty second instar $P$. xylostella larvae were placed in the Petri dish and mortalities were recorded after $72 \mathrm{~h}$. Each test was performed in triplicate.

Supporting Information The ${ }^{1} \mathrm{H}$ NMR, ${ }^{13} \mathrm{C}$ NMR and ${ }^{19} \mathrm{~F}$ NMR spectra of compounds $\mathbf{6 a} \sim \mathbf{6} \mathbf{a b}$. The Supporting Information is available free of charge via the Internet at http://sioc-journal.cn.

\section{References}

[1] Oerke, E. C. J. Agric. Sci. 2006, 144, 31.

[2] Carvalho, F. P. Food Energy Secur. 2017, 6, 48.

[3] Xiang, Y. J.; Fu, H. J.; Li, Q. Y.; Li, X. Y.; N, J. Y.; Zhou, X. G.; Li, H. H.; Zhang, H. J. Mount. Agric. Biol. 2019, 38, 46 (in Chinese). (向运佳, 符慧娟, 李其勇, 李星月, 倪建英, 周小刚, 李洪浩, 张鸿，山地农业生物学报, 2019, 38, 46.)

[4] Huang, N.; Angeles, E. R.; Domingo, J.; Magpantay, G.; Singh, S.; Zhang, G.; Kumaravadivel, N.; Bennett, J.; Khush, G. S. Theor. Appl. Genet. 1997, 95, 313.

[5] Srinivasan, B. Curr. Sci. 2005, 88, 1229.

[6] Li, P.; Tian, P. Y.; Chen, Y. Z.; Song, X. P.; Xue, W.; Jin, L. H.; Hu, D. Y.; Yang, S.; Song, B. Pest Manage. Sci. 2018, 74, 844.

[7] Maxwell, T. J.; Rajasekaran, P.; Das, S.; Campos, M. G. N.; Young, M.; Mendis, H. C.; Ozcan, A.; Gerberich, K. M.; Myers, M. E.; Graham, J. H.; Johnson, E. G.; Santra, S. J. Agric. Food Chem. 2019, 67, 12393.

[8] Gottwald, T. R.; Hughes, G.; Graham, J. H.; Sun, X. A.; Riley, T. Phytopathology 2001, 91, 30.

[9] Das, A. K. J. Appl. Hortic. 2003, 5, 52.

[10] Zhang, H.; Li, J. X.; Chen, S. Y.; Ding, H. X.; Yu, S.; Peng, L. J. J. Guizhou Univ. (Nat. Sci. Ed.) 2019, 36, 33 (in Chinese). (张恒, 李金星, 陈思颖, 丁海霞, 余水, 彭丽娟, 贵州大学学报 (自然科学版) , 2019, 36, 33.)

[11] Kemmitt, G.; Sparks, T. C.; Hunter, J. E.; Lorsbach, B. A.; Hanger, G.; Gast, R. E.; Bryant, R. J. J. Agric. Food Chem. 2018, 66, 10337.

[12] Zhang, Y.; Pan, X. Y.; Duan, Y. B.; Zhu, X. F.; Ma, X. W.; Huang, T. T.; Gao, T. C.; Zhou, M. G. Australas. Plant Pathol. 2015, 44, 541.

[13] Behlau, F.; Canteros, B. I.; Jones, J. B.; Graham, J. H. Eur. J. Plant Pathol. 2012, 133, 949.

[14] Lang, J. M.; DuCharme, E.; Caballero, J. I.; Luna, E.; Hartman, T.; Ortiz-Castro, M.; Korus, K. A.; Rascoe, J.; Jackson-Ziems, T. A.; Broders, K.; Leach, J. E. Phytopathology 2017, 107, 1312.

[15] Burriss, A.; Edmunds, A. J. F.; Emery, D.; Hall, R. G.; Jacob, O.; Schaetzer, J. Pest Manage. Sci. 2018, 74, 1228.

[16] Cao, S.; Qian, X. H.; Song, G. H.; Huang, Q. C. J. Fluorine Chem. 2002, 117, 63 .

[17] Wang, F.; Tang, P. P. Chin. J. Org. Chem. 2020, 40, 1805 (in Chinese). (王丰, 汤平平, 有机化学, 2002, 40, 1805.)

[18] Kirby, N. V.; Daeuble, J. F.; Davis, L. N.; Hannum, A. C.; Hellwig, K.; Lawler, L. K.; Parker, M. H.; Pieczko, M. E. Pest Manage. Sci. 2001, 57, 844 .

[19] Yang, G. Z.; Zhu, J. K.; Yin, X. D.; Yan, Y. F.; Wang, Y. L.; Shang, X. F.; Liu, Y. Q.; Zhao, Z. M.; Peng, J. W.; Liu, H. J. Agric. Food Chem. 2019, ${ }_{6} 7,11340$

[20] Hua, X. W.; Liu, N. N.; Fan, Z. J.; Zong, G. G.; Ma, Y.; Lei, K.; Yin, H.; Wang, G. Q. Chin. J. Org. Chem. 2019, 39, 2581 (in Chinese).

(华学文, 刘南南, 范志金, 宗广宁，马翼，雷康，殷昊，王桂清， 有机化学, 2019, 39, 2581.)

[21] Wang, Y. Y.; Xu, F. Z.; Luo, D. X.; Guo, S. X.; He, F.; Dai, A. L.; Song, B. A.; Wu, J. J. Agric. Food Chem. 2019, 67, 13344.

[22] Nugent, B. M.; Buysse, A. M.; Loso, M. R.; Babcock, J. M.; John- 
son, T. C.; Oliver, M. P.; Martin, T. P.; Ober, M. S.; Breaux, N.; Robinsona, A.; Adelfinskaya, Y. Pest Manage. Sci. 2015, 71, 928.

[23] Cao, S.; Qian, X. H.; Song, G. H.; Huang, Q. C. J. Fluorine Chem. 2002, 117, 63

[24] Cai, M. Y.; Li, Z.; Fan, F.; Huang, Q. C.; Shao, X. S.; Song, G. H. J. Agric. Food Chem. 2010, 58, 2624.

[25] Chen, J. X.; Yi, C. F.; Wang, S. B.; Wu, S. K.; Li, S. Y.; Hu, D. Y.; Song, B. A. Bioorg. Med. Chem. Lett. 2019, 29, 1203.

[26] Dai, A. L., Guo, S. X.; Li, C. H.; Zhang, R. F.; Wu, J. Modern Agrochem. 2020, 19, 21 (in Chinese).

(代阿丽，郭声金金，李传会，张仁风，吴剑，现代农药， 2020，19， 21.)

[27] Deng, H. X. Zhejiang Chem. Ind. 2011, 42, 1 (in Chinese). (邓红霞, 浙江化工, 2011, 42, 1.)

[28] Aggarwal, N.; Kumar, R.; Srivastva, C.; Dureja, P.; Khurana, J. M. J. Agric. Food Chem. 2010, 58, 3056.

[29] Du, H.; Fan, Z. J.; Yang, L.; Bao, X. P. Chin. J. Org. Chem. 2018, 38,531 (in Chinese).

(杜欢，范治江，杨岗，鲍小平，有机化学, 2018, 38, 531.)

[30] Hong, Y.; Dai, H.; Ye, L. Y.; Zhong, S. L.; Gao, X. F.; Shi, Y. J.; Li, C. J.; Shi, J.; Shi, L. Chin. J. Org. Chem. 2017, 37, 3006 (in Chinese).

(洪宇, 戴红, 叶林玉, 仲苏林, 曹雄飞, 石玉军, 李春建, 石健, 有机化学, 2017, 37, 3006.)

[31] Luo, D. X.; Guo, S. X.; He, F.; Chen, S. X.; Dai, A. L.; Zhang, R. F.; Wu, J. J. Agric. Food Chem. 2020, 68, 7226.

[32] Chen, Y.; Li, P.; Chen, M.; Su, S. J.; He, J.; Zhang, M. Liu, L. W.;
Xue, W. Chin. J. Org. Chem. 2019, 39, 2813 (in Chinese) (陈英, 李普, 陈梅, 苏时军, 贺军, 张敏, 柳立伟, 薛伟, 有机 化学, 2019, 39, 2813.)

[33] Shao, L. H.; Gan, Y. Y.; Hou, M.; Tao, S. L.; Zhang, L. Q.; Wang, Z. C.; Ouyang, G. P. Chin. J. Org. Chem. 2020, 40, 1975 (in Chinese).

(邵利辉，甘宜远，侯迷，陶世林，张丽琼，王贞超，欧阳贵平， 有机化学, 2020, 40, 1975.)

[34] Xu, F. Z.; Wang, Y. Y.; Luo, D. X.; Yu, G.; Wu, Y. K.; Dai, A. L.; Zhao, Y. H.; Wu, J. ChemistrySelect 2018, 3, 2795.

[35] Kang, S. H.; Song, B. A.; Wu, J.; He, M.; Hu, D. Y.; Jin, L. H.; Zeng, S.; Xue, W.; Yang, S. Eur. J. Med. Chem. 2013, 67, 14.

[36] Li, P.; Hu, D. Y.; Xie, D. D.; Chen, J. X.; Jin, L. H.; Song, B. A. J. Agric. Food Chem. 2018, 66, 3093.

[37] Dalgaard, P.; Ross, T.; Kamperman, L.; Neumeyer, K.; McMeekin, T. A. Int. J. Food Microbiol. 1994, 23, 391.

[38] Lungu, L.; Ciocarlan, A.; Barba, A.; Shova, S.; Pogrebnoi, S.; Mangalagui, L.; Moldoveanu, C.; Vornicu, N.; Ambrosio, M. D.; Babak, M. V.; Arion, V. B.; Aricu, A. Chem. Heterocycl. Compd. 2019, 55,716 .

[39] Maienfisch, P.; Hall, R. G. Chimia 2004, 58, 93.

[40] Theodoridis, G. Adv. Fluorine Sci. 2006, 2, 121.

[41] Feng, M. L.; Li, Y. F.; Zhu, H. J.; Zhao, L. Xi, B. B.; Ni, J. P. J. Agric. Food Chem. 2010, 58, 10999.

[42] Abbott, W. S. J. Econ. Entomol. 1925, 18, 265.

[43] Zhao, Q. Q.; Li, Y. Q.; Xiong, L. X.; Wang, Q. M. J. Agric. Food Chem. 2010, 58, 4992. 